\title{
"Et l'homme tout entier devient dieu": La déification selon Origène à la lumière des nouvelles Homélies sur les Psaumes*
}

\author{
Pier Cesare Bori
}

IN MEMORIAM

\author{
Lorenzo Perrone \\ "ALMA MATER STUDIORUM" - UNIVERSITÀ DI BOLOGNA \\ DIPARTIMENTO DI FILOLOGIA CLASSICA E ITALIANISTICA \\ lorenzo.perrone@unibo.it
}

Précis: L'article se propose de revisiter le thème de la déification chez Origène à la lumière de plusieurs perspectives: lexique, références scripturaires, aspects exégétiques et théologiques. La doctrine origénienne se manifeste sous plusieurs facettes. La déification s'articule á l'intérieur d'un discours biblique et sotériologique. La divinisation de l'homme, engageant aussi son corps selon le modèle archétypique de Christ ressucité, débouche finalement sur l'immortalité et l'incorruption retrouvées autant que sur la pleine acquisition de la condition de fils adoptifs de Dieu.

Mots-clés: déification, Origène, soteriologie, rédemption, glorification.

Astract: This paper reviews the topic deification according to Origen in lexicographical, scriptural references, exegetical and theological aspects. Origen's doctrine on deification is rich, has its place as part of a biblical and soteriological view. Includes also the body according to the risen Christ as archeypical model, leads in the end to immortality, incorruption and to the full condition of adopted children of God.

Key words: deification, Origen, soteriology, ransom, glorification.

* Exposé presenté en occasion du colloque «Exégèse, révélation et formation des dogmes dans l'Antiquitè tardive» (EPHE Paris, 25-26 octobre 2013). Je remercie cordialement Alain Le Boulluec pour la patiente révision de mon texte français. 
I. LE THÈME DE LA DÉIFICATION À LA CROISÉE DES DISCOURS THÉOlOGIQUES ET PHILOSOPHIQUES

Quand on évoque la notion de 'divinisation' ou 'déification' dans l'histoire de la pensée chrétienne, généralement on pense d'abord à la théologie byzantine. Selon l'avis de nombreux spécialistes, c'est à elle que reviendraient les développements les plus importants sur ce thème de la 'déification' de l'homme, l'élaboration la plus célèbre étant celle offerte par Grégoire Palamas et les théologiens palamites ${ }^{1}$. Toutefois, l'essor théologique qui se produisit à ce propos vers la fin de l'époque byzantine venait de loin, puisqu'il avait des racines assez anciennes, et non seulement dans les auteurs de la patristique grecque tardive, comme par exemple Maxime le Confesseur ${ }^{2}$. On pourrait remonter bien plus en arrière dans le temps, mettant en lumière les présupposés pour le discours théologique sur la déification qui sont déjà ébauchés dans les Écritures mêmes, soit l'Ancien soit le Nouveau Testament, et prendre entre autres Paul comme témoin privilégié3. En plus, il faudrait réfléchir sur le fait que l'idée biblique de la destinée spirituelle de l'homme comme marquée par la vocation à 'se rendre semblable' à Dieu n'était pas du tout étrangère à la philosophie ancienne; en particulier, comme

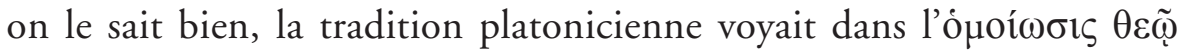
l'accomplissement transcendant de l'homme, réalisant ainsi son 'affinité'

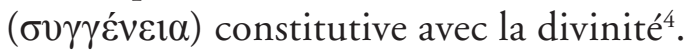

1. Voir, par ex., J. Meyendorff, Byzantine Theology. Historical Trends and Doctrinal Themes, New York, $1979^{2}$. J'ai analysé cette perspective dans mon introduction à la traduction italienne: L. Perrone, "Teofania nell'uomo. Il messaggio della teologia bizantina secondo J. Meyendorff», dans J. Meyendorff, La teologia bizantina. Sviluppi storici e temi dottrinali, tr. it. Casale Monferrato, 1984, p. xi-xxvii.

2. Pour une vue d'ensemble, cf. N. Russell, The Doctrine of Deification in the Greek Patristic Tradition, Oxford, 2004. Voir aussi plus récemment D.V. Meconi, The One Christ: St. Augustine's Theology of Deification, Washington/DC, 2013.

3. Selon la toute nouvelle analyse de M.D. Litwa, We Are Being Transformed. Deification in Paul's Soteriology, Berlin-New York, 2012.

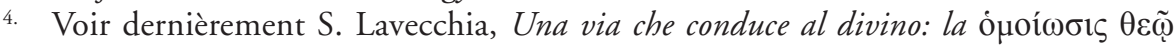
nella filosofia di Platone, Milano, 2006. Le texte principal de Platon est Theaet. 176b, cité par Origène, Prin III,6,1: summum bonum sit, prout possibile est, similem fieri deo. Sur sa fortune patristique, cf. la monographie classique: H. Merki,

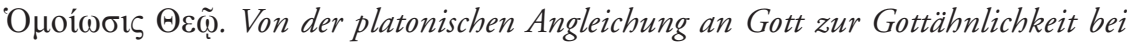
Gregor von Nyssa, Fribourg, 1952. Concernant les multiples résonnances du motif de la 'ressemblance' à Dieu, voir In the Image of God. Foundations and Objections within the Discourse on Human Dignity. Proceedings of the Colloquium at Bologna 
Ce tableau tout à fait sommaire, impliquant par ailleurs plusieurs approches du point de vue historique et doctrinal, nous invite à situer d'abord l'apport donné par Origène au discours sur la déification dans une tradition de pensée qui s'avère être si riche. Il s'agit sans doute d'une contribution décisive, bien qu'elle ait été contestée et en partie oubliée par la suite, en raison de sa condamnation pour hétérodoxie. Car l'Alexandrin aborde notre thème dans son horizon le plus vaste: premièrement, il l'énonce au niveau du langage, grâce à l'emploi d'une riche terminologie plus ou moins 'technique', qui anticipe largement les usages postérieurs. En deuxième lieu, il rassemble un dossier scripturaire très abondant, au sein duquel nous pouvons observer la présence d'éléments constants ainsi que des combinaisons différentes de textes. Enfin, il inscrit le motif de la déification, de façon explicite ou implicite, dans tout l'ensemble de son 'système' théologique, allant ainsi de la doctrine de la préexistence jusqu'à celle de l'apocatastase. Cela le pousse par conséquent à lui attribuer un relief structurel, quoiqu'il refaçonne ses énoncés à plusieurs égards et les représente, pour ainsi dire, sous des vêtements divers ${ }^{5}$. Nous aurons la chance de le constater non seulement à travers l'analyse des écrits d'Origène déjà connus, mais spécialement à la lumière du nouveau corpus des 29 Homélies sur les Psaumes découvert récemment à Munich par Marina Molin Pradel ${ }^{6}$. Comme nous le verrons bientôt, ces sermons confirment abondamment la perspective

and Rossena (July 2009) in Honour of Pier Cesare Bori, ed. by A. Melloni and R. Saccenti, Berlin, 2010.

5. P. Martens, Art. "Divinization", in The Westminster Handbook to Origen, ed. by J.A. McGuckin, Louisville-London, 2004, 91-93 souligne à juste titre la portée structurelle du motif de la 'déification' chez Origène. L'ouvrage classique sur notre thème reste J. Rius-Camps, El dinamismo trinitario en la divinización de los seres racionales según Orígenes, Roma, 1970.

6. Sur cette découverte exceptionnelle je me permets de renvoyer aux contributions parues jusqu’à présent ou en cours de parution: M. Molin Pradel, «Novità origeniane dalla Staatsbibliothek di Monaco di Baviera: il Cod. graec. 314", Adamantius, 18, 2012, p. 16-40; L. Perrone, "Riscoprire Origene oggi: prime impressioni sulla raccolta di omelie sui salmi nel Codex Monacensis Graecus 314», Adamantius, 18, 2012, p. 41-58; "Rediscovering Origen Today: First Impressions of the Newly Discovered Collection of Homilies on the Psalms», in Studia Patristica LVI/4, ed. by M. Vinzent, Leuven, 2013, p. 103-122; "Origenes rediuiuus: La découverte des homélies sur les Psaumes dans le Cod. Gr. 314 de Munich", Revue des Études Augustiniennes et Patristiques, 59, 2013, p. 55-93. 
que nous venons d'évoquer, tout en conférant au thème origénien de la déification une intensité remarquable.

\section{LE LANGAGE ORIGÉNIEN DE LA 'DÉIFICATION': QUELQUES APERÇUS TERMINOLOGIQUES}

Ayant conscience de la complexité de notre thème, il est peut-être utile de l'introduire par quelques aperçus à propos de la terminologie. Comme on l'aura remarqué, nous avons fait recours alternativement aux termes 'divinisation' ou 'déification', sans les distinguer autrement. Notre emploi laisse donc deviner un usage synonymique, ce qui est légitime, au moins sous certains points de vue, mais demande quand même des précisions. Si nous regardons de près le vocabulaire d'Origène, nous y retrouvons des expressions qui formulent l'idée de la 'déification' sans la lier cependant à des termes techniques trop étroits, comme il arrive souvent chez lui. L'Alexandrin se sert de préférence de la forme passive du verbe $\theta \varepsilon o \pi$ olé $\omega$, tout en l'utilisant aussi (surtout à l'actif) avec une signification négative, lorsqu'il s'agit de dénoncer les faux dieux du paganisme ou de rejeter la création de nouvelles idoles ou même d'appliquer par une exégèse allégorique aux hérétiques la 'divinisation' que les Samaritains sont censés faire du Mont Garizim ${ }^{7}$ Ces mêmes hérétiques, selon la complexe allégorie

\footnotetext{
La forme active de $\theta \varepsilon$ coøoı́́ $\omega$ est attestée par HIer $\mathrm{V}, 2$, où Origène critique la divinisation des réalités matérielles, en premier lieu l'idole de l'argent: ov̉oćva

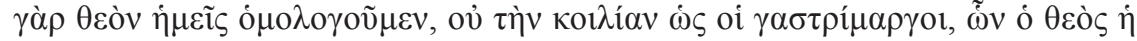

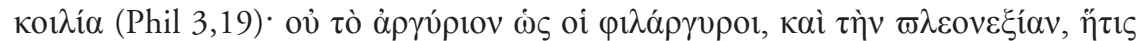

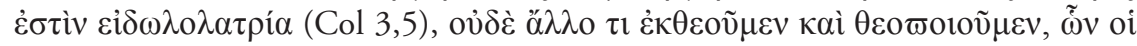

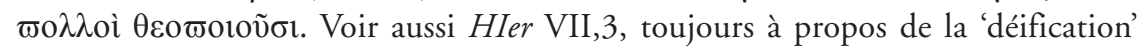

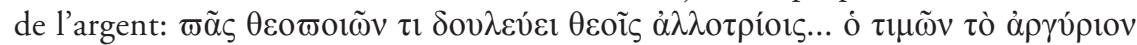

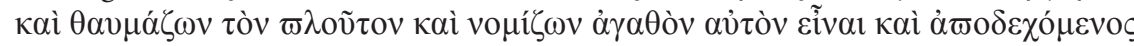

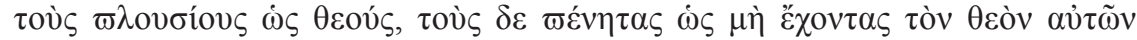

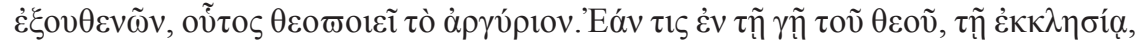

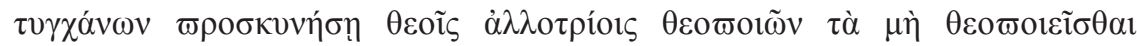

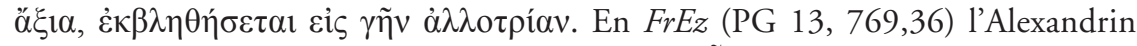

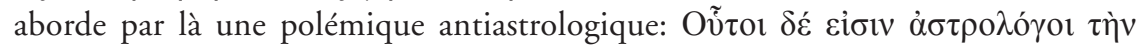

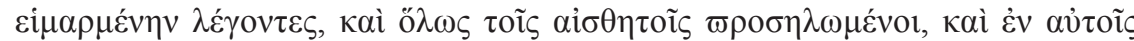

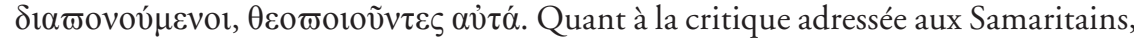

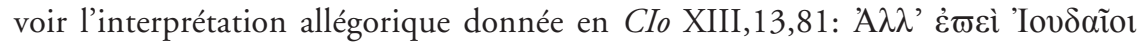

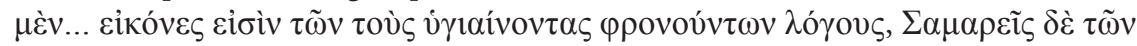

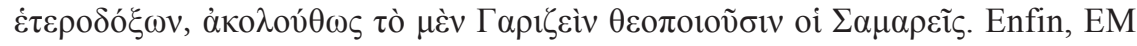
7 explique la défense de jurer 'par le ciel' ou 'par la terre' donnée par Jésus
} 
de la loi du lévirat donnée par Origène dans le 17ème Livre du Commentaire sur Matthieu, se refusent d'être divinisés' en apportant des fruits ${ }^{8}$. Au sens positif comme ici, la forme passive de $\theta \varepsilon 0 \varpi 0 เ \varepsilon ́ \omega$ - quoique par un emploi bien plus restreint par rapport à sa prédominante qualification négative ${ }^{9}$ - caractérise le processus de la 'déification' dans plusieurs passages des ouvrages d'Origène, dont les plus importants se trouvent dans le Commentaire sur Jean et le Traité sur la prière.

Considérant d'abord les occurrences de nature plus générique, nous en signalons une dans l'Exhortation au martyre à propos des martyrs macchabéens, persécutés par "l'Adversaire de Dieu qui poursuit ceux qui ont été divinisés par le Logos". Ce passage laisse pressentir la divinisation comme un processus en acte dans celui qui s'achemine sur la voie de la sainteté ou de la perfection avec le soutien du $\operatorname{Logos}^{10}$. Une énonciation semblable d'ordre général, mais plus circonstanciée et plus décisive à propos de la dynamique trinitaire de ce processus, revient dans le 2ème Livre du Commentaire sur Jean. Il s'agit du lieu célèbre autant que controversé, où Origène distingue le Père en tant que ó $\theta \varepsilon$ ćs, Dieu au sens propre, du Logos comme $\theta \varepsilon$ ó $\zeta$ sans l'article, c'est-à-dire comme participant à titre suréminent de la divinité du Père (en tant que premier des êtres 'divinisés' - $\theta \varepsilon$ øo comme son ministre pour la déification des êtres inférieurs, eux aussi à regarder comme des 'dieux' ( $\theta \varepsilon$ oí) grâce à l'action divinisatrice du Lo-

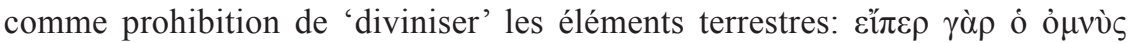

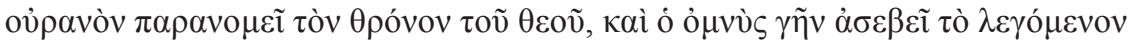

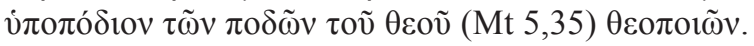

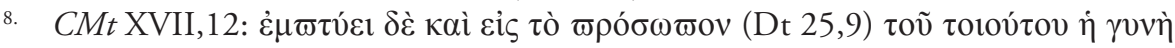

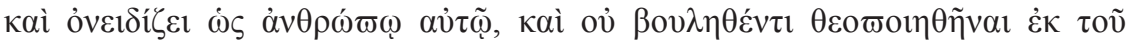

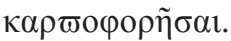

9. L'emploi négatif du passif de $\theta \varepsilon 0 \varpi 01 \varepsilon ́ \omega$ figure dans $C M t \mathrm{XI}, 12$, où l'on retrouve à

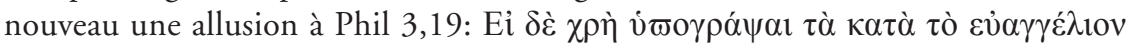

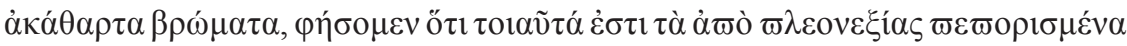

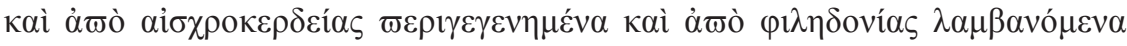

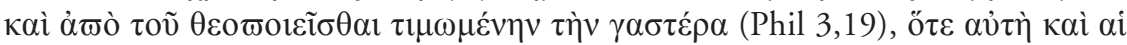

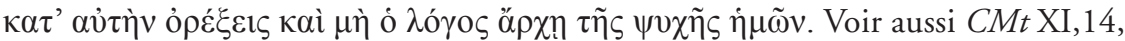

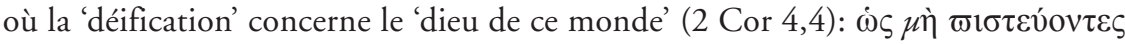

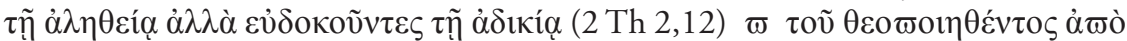

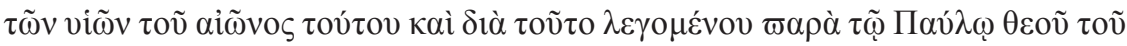

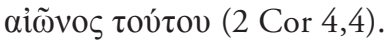

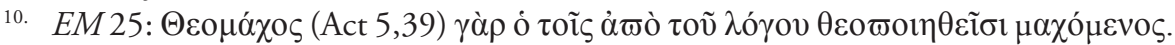


gos $^{11}$. Ce texte fondamental, dont nous allons nous occuper à nouveau par la suite, énonce le thème de la déification, non seulement en dessinant acteurs et facteurs de ce processus, mais il l'articule aussi par le biais

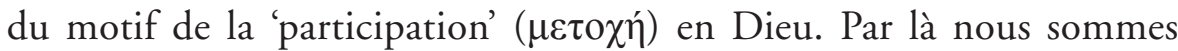
renvoyés à un horizon linguistique et conceptuel plus vaste que la termi-

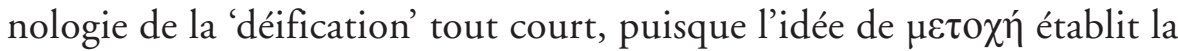
relation ontologique entre l'être absolu de Dieu et les êtres dépendants de lui, quoique cette dépendance puisse se donner de façon diversifiée ${ }^{12}$.

Revenons à $\theta \varepsilon 0 \varpi 01 \varepsilon i ̃ \sigma \theta \alpha$. Dans le 32ème Livre du Commentaire sur Jean l'Alexandrin réfléchit sur la notion de 'gloire de Dieu', en commentant Jn 13,31-32 ("Quand il fut sorti, Jésus dit: 'Maintenant le Fils de l'homme a été glorifié et Dieu a été glorifié en lui. Si Dieu a été glorifié en lui, Dieu aussi le glorifiera en lui-même et il le glorifiera bientôt"). Il ne s'agit pas en premier lieu, pour l'exégète, de la 'gloire' comme l'entendent les philosophes, c'est-à-dire en tant que "louange de la part de beaucoup". Il s'efforce donc de l'éclairer en recourant à des passages scripturaires tirés de l'Ancien et du Nouveau Testament et considérés

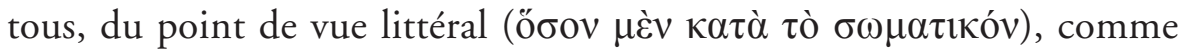
des épiphanies divines: d'abord, pour les textes vétéro-testamentaires, en rapport à la gloire divine qui remplit la Tente du Témoignage selon le récit de l'Exode (Ex 40,34-35) ou la maison du Seigneur dans le 3ème

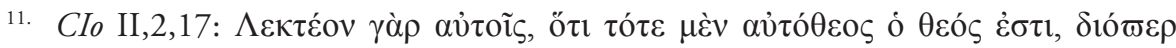

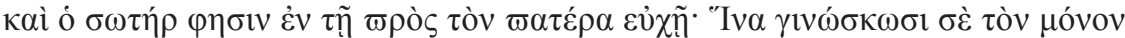

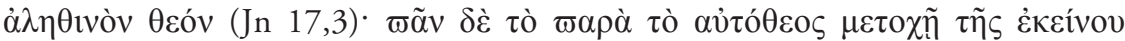

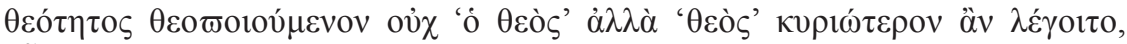

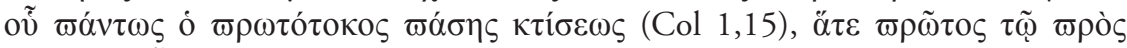

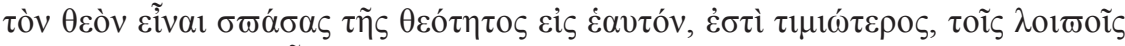

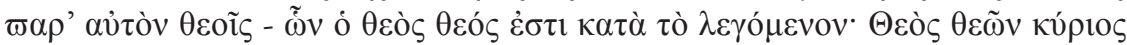

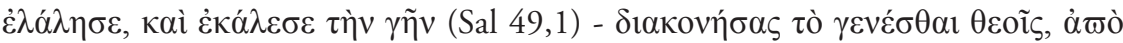

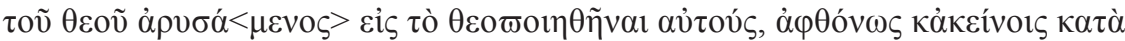

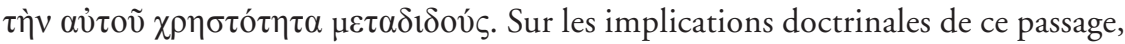
cf. dernièrement C. Bruns, Trinität und Kosmos. Zur Gotteslehre des Origenes, Münster, 2013, spécialement p. 275-277.

12. Cf. D. Balas, «The Idea of Participation in the Structure of Origen's Thought. Christian Transposition of a Theme of the Platonic Tradition", in Origeniana. Premier colloque international des études origéniennes, dir. par H. Crouzel, G. Lomiento, J. Rius-Camps, Bari, 1975, p. 257-275. Sur les prémisses philosophiques et les développements patristiques, voir maintenant T.T. Tollefsen, Activity and Participation in Late Antique and Early Christian Thought, Oxford 2012. Par ailleurs, il ne traite que marginalement la pensée d'Origène. 
Livre des Règnes (3 Rg 8,10-11), ou encore celle qui resplendissait sur le visage de Moïse au moment où il descendait du Sinaï après sa conversation avec Dieu (Ex 34, 29-30). Ensuite, passant au Nouveau Testament, il rappelle la gloire du Christ dans l'épisode de la Transfiguration (Lc 9,29-31), ainsi que la reprise du récit de la gloire de Moïse au Sinaï que fait Paul dans la 2ème Épître au Corinthiens (2 Cor 3,18). C'est ce dernier passage qui attire spécifiquement le motif de la déification: par une

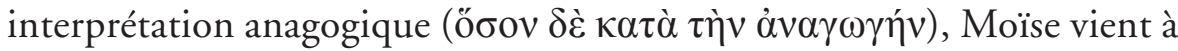
symboliser l'ascension vers Dieu de l'intellect purifié de ses attaches aux réalités sensibles pour s'adonner à la contemplation de Dieu; or, cette contemplation apporte à l'intellect la 'déification'13.

Les deux passages contenus dans le Perì euchês relient à leur tour notre thème au discours des 'nourritures spirituelles', qui conjointement à l'idée de la 'participation' nous aide à mieux comprendre les modalités de la 'divinisation' de l'homme envisagée par Origène. L'agent du processus est toujours identifié dans le Logos (ce qui pose évidemment encore une fois le problème du rôle du Saint Esprit, par ailleurs reconnu clairement par l'Alexandrin dans le Perì archôn comme le principe de la sanctification). Résumant l'explication qu'il a donnée des deux premières demandes du Notre Père, Origène exhorte à prier "sans cesse" (1 Th 5,17) dans une disposition d'esprit "déifiée par le Logos", en vue de parvenir au moment où "Dieu sera tout en tous" (1 Cor 15,28) ${ }^{14}$. Ensuite, au terme du commentaire consacré au "pain supersubstantiel”

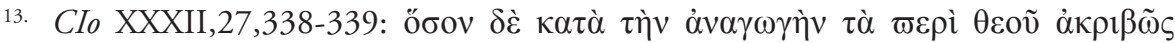

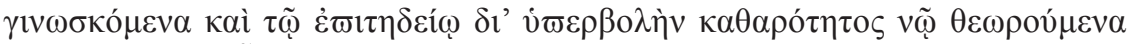

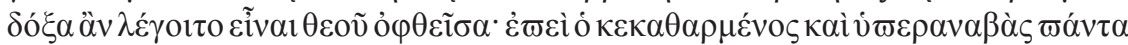

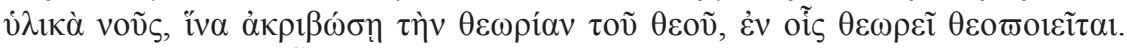

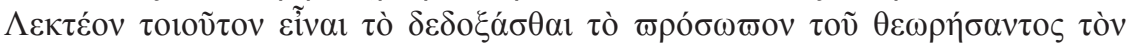

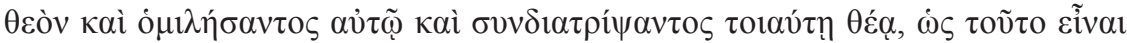

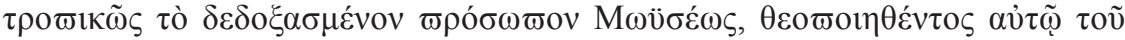
voṽ. Sur ce passage voir H. Crouzel, Théologie de l'image de Dieu chez Origène, Paris, 1956, p. 232ss. Quant à l'interprétation origénienne de 2 Cor 3,18, cf. Id., Origène et la "connaissance mystique", Paris, 1961, p. 118.

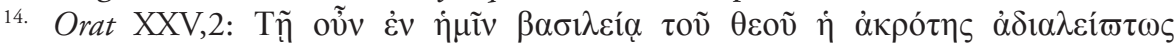

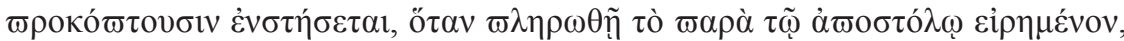

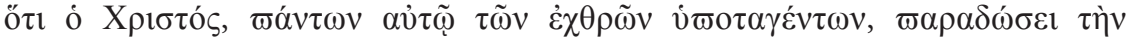

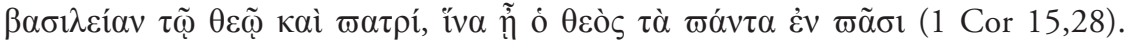

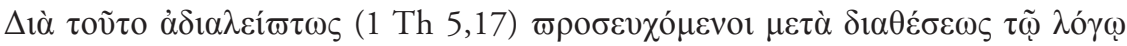

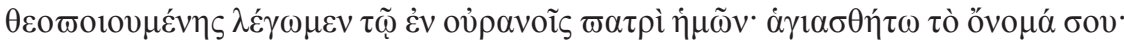
$\dot{\varepsilon} \lambda \theta \varepsilon \dot{\tau} \omega \dot{\eta} \beta \alpha \sigma \imath \lambda \varepsilon i ́ \alpha$ бov (Mt 6,9-10; Lc 11,2). 
dans la quatrième demande de la Prière du Seigneur, adoptant à nouveau un mode exhortatif, il souligne la nécessité de prier pour être nourri par ce 'pain', qui est Dieu, le Logos 'dans le principe' et 'auprès de Dieu' (Jn 1,1) afin d'être déifié par lui ${ }^{15}$.

À notre dossier terminologique, établi jusqu'ici à la lumière des écrits déjà connus d'Origène, il faut maintenant ajouter l'apport des Homélies sur les Psaumes dans le Codex Graecus 314 de Munich. Elles confirment le tableau que nous venons d'illustrer, y apportant d'autre part l'unique

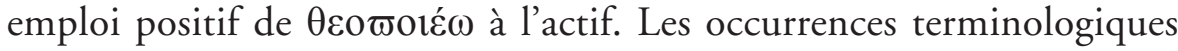
se repèrent toutes dans l'Homélie sur le Psaume 81, consacrée spécifiquement à notre thème. Elle enrichit le discours origénien sur la déification - comme nous le reverrons tout à l'heure - par rapport non plus aux agents et facteurs, mais plutôt à son objet. Ceux-ci ne se limitent pas à l'intellect' (voṽs), ainsi qu'on l'a vu précédemment, mais ils incluent l'homme dans toutes ses composantes, conformément à l'anthropologie tripartite de l'Alexandrin. À ce propos, il remarque que le fait de 'diviniser' l'esprit' ( $ఐ v \varepsilon \tilde{v} \mu \alpha)$ ne nous surprend pas, étant donné l'affinité de l'homme avec Dieu, dont le siège premier et plus approprié demeure précisément dans l'esprit' de l'homme. Plus surprenant est sans doute, au dire d'Origène, le fait que l'âme' ( $\psi v \chi \eta ̛$ ) soit elle aussi 'déifiée', étant donné qu' elle constitue le lieu instable du libre arbitre, mais ce qui nous surprend plus que tout autre chose est la 'déification' du corps ${ }^{16}$. Le sujet sous-entendu de cet emploi à l'actif, celui qui agit en conférant la 'divi-

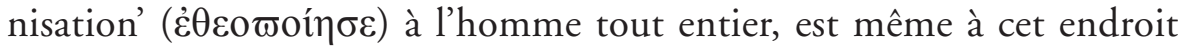
Dieu le Verbe, comme on peut le dégager du contexte proche.

Si le texte de l'Homélie sur le Psaume 81 se distingue - au niveau de l'énonciation terminologique - par une formulation bien plus immé-

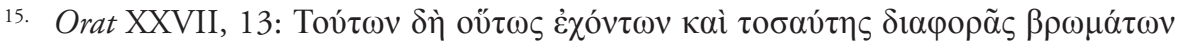

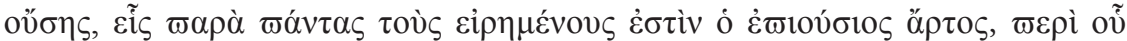

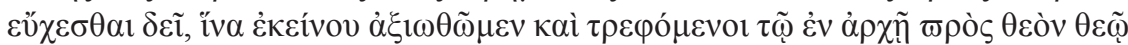

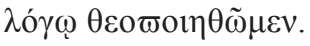

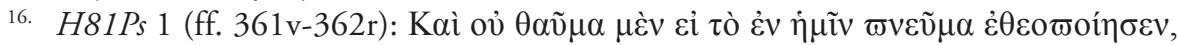

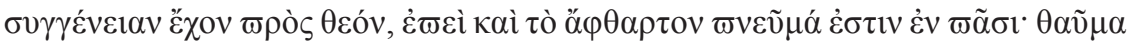

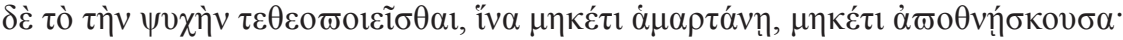

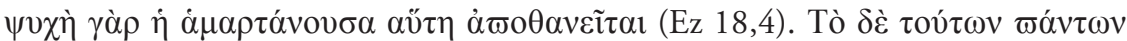

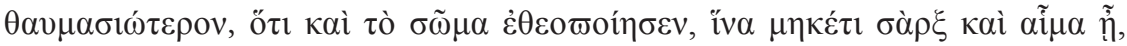

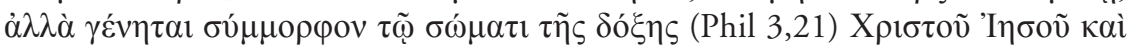

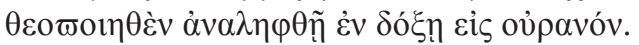


diate et concrète que les autres données de vocabulaire, nous en retrouvons une autre semblable dans le 2ème Homélie sur le Psaume 76. En effet, Origène y évoque les patriarches Abraham, Jacob et Isaac en les appellant chacun 'dieu' $(\theta \varepsilon o ́ \varsigma)$, en raison de leur association au nom divin en Ex 3,6. Comme l'indique ce passage scripturaire, ils sont des

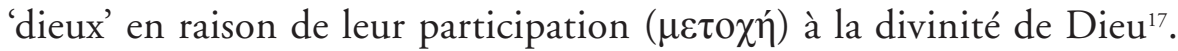
Nous nous retrouvons donc face à des personnages bibliques, qu'Origène n'hésite pas à regarder comme des 'dieux'. Évidemment, ils n'ont pas l'exclusivité d'une telle appellation, comme le montre la suite de l'homélie. Elle reprend en pratique les idées du 2ème Livre du Commentaire sur Jean (CIo II,2,17): l'Alexandrin, après avoir rappelé le Logos en tant que 'Dieu' $\left(\theta \varepsilon \delta \delta_{\zeta}\right)$, observe que si on doit considérer les 'justes' des 'dieux', à plus forte raison faut-il le dire à propos des 'anges'. Nous reviendrons bientôt sur ce point dans l'analyse des lieux scripturaires qui soutiennent le discours origénien sur la déification.

Pour compléter le relevé lexical autour de $\theta \varepsilon$ cooté $\omega$, il faut enfin considérer le terme $\theta \varepsilon 0 \varpi 0$ ó $\varsigma$, "qui fait dieu”. Attesté jusqu’à présent dans un fragment sur Ézéchiel, il revient maintenant aussi dans l'Homélie sur le Psaume 81. Comme le suggère sa signification immédiate, le mot exprime l'agent ou le facteur de 'divinisation'. Dans les deux passages, il ne peut donc que viser l'action du Logos 'divinisateur', le fragment sur Ézéchiel étant en ce sens plus spécifique. Il concerne, en effet, la vocation prophétique d'Ézéchiel, qui par effet de l'inspiration a lui aussi titre - quoique de façon implicite grâce à la citation de Ps 81,6 - à être rangé, dirions-nous, parmi les 'dieux'. Surtout, comme le précise notre passage, le Verbe qui se donne à Ézéchiel est Dieu en tant que Logos "qui fait dieu" $(\theta \varepsilon 0 \varpi 010 ́ \varsigma)^{18}$. Ce passage s'avère un concentré

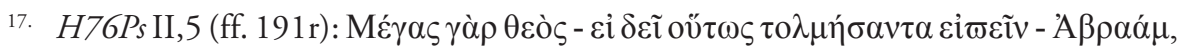

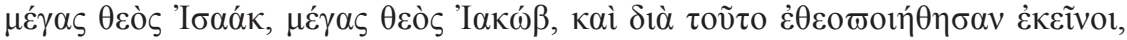

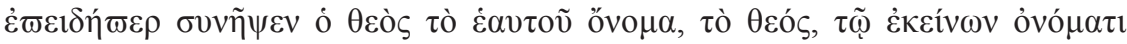

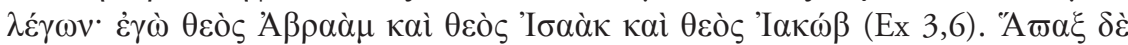

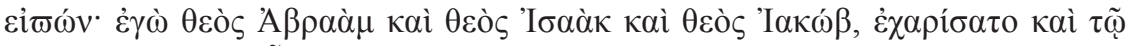

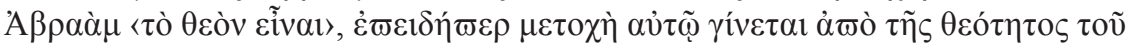

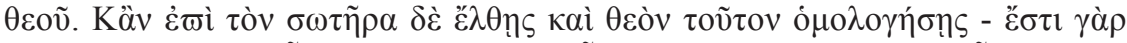

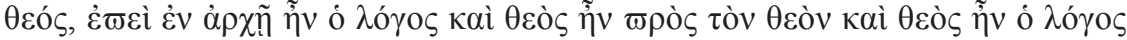

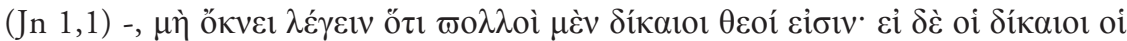

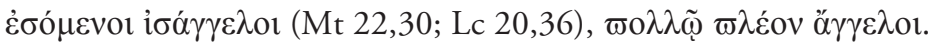

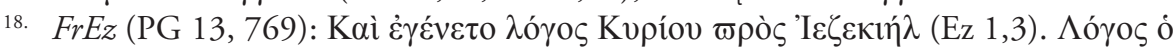

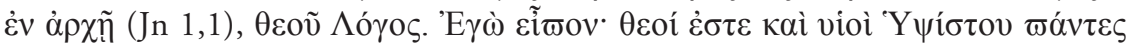


scripturaire, du fait qu'il réunit par un dossier essentiel quelques-uns des testimonia les plus classiques pour la réflexion d'Origène. Sur un fondement analogue, l'Homélie sur le Psaume 81 généralise l'énoncé des Selecta in Ezechielem, en soutenant d'abord le principe selon lequel, quand le Verbe vient à l'âme, il la divinise. Par conséquent, l'action du Logos pour déifier les trois composantes de l'homme suivant l'argumentation que nous avons retracée auparavant -, opère en lui comme un 'levain' incomparable ${ }^{19}$.

Les autres supports lexicaux du langage de la déification ne modifient pas substantiellement nos résultats. Il faut néanmoins les mentionner eux-aussi, car Origène s'en sert de façon complémentaire, en l'occurrence

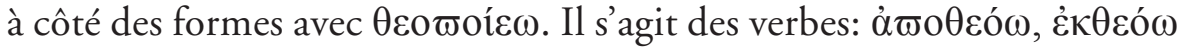

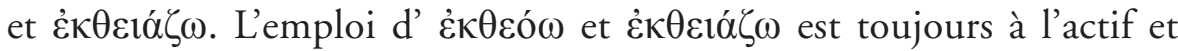
pour la plupart au sens négatif, c'est-à-dire pour stigmatiser les formes inacceptables de 'divinisation': par exemple, dans la 2ème Homélie sur le

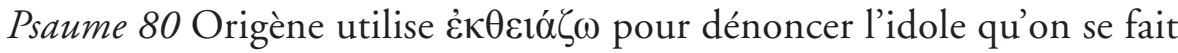

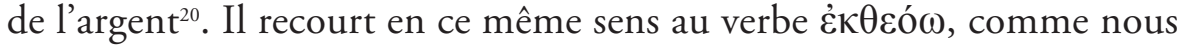
le voyons dans la 5ème Homélie sur Jérémie (en liaison même ici avec Col $3,5)^{21}$. D'autre part l'Alexandrin, dans le 10ème Livre du Commentaire

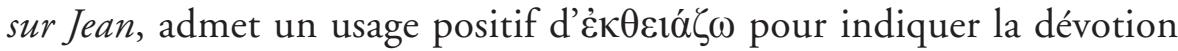
au Temple de Jérusalem de la part des Juifs ainsi que de Jésus et ses disciples, en tant que lieu 'divinisé' à la suite du culte rendu à Dieu ${ }^{22}$.

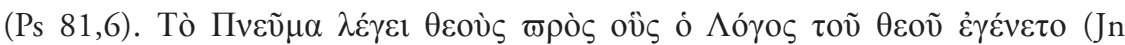

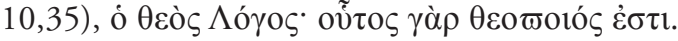

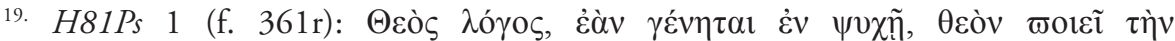

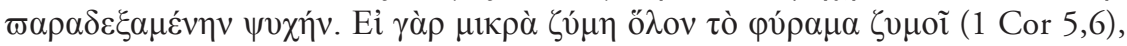

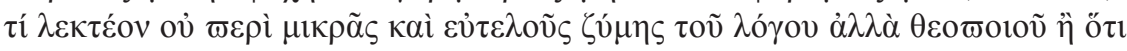

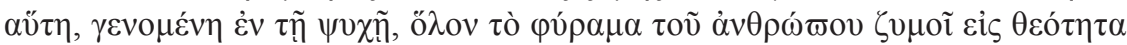

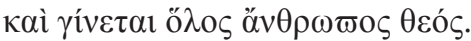

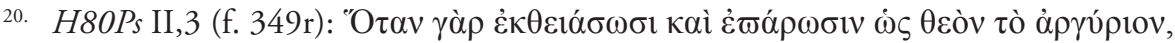

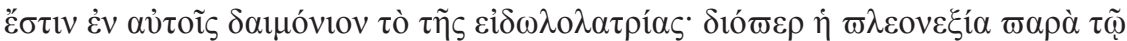

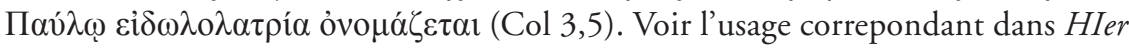

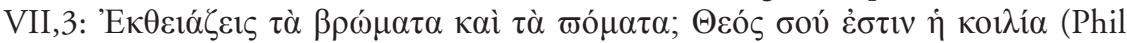

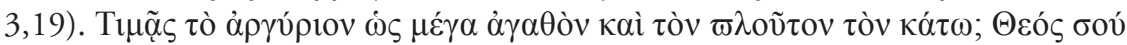

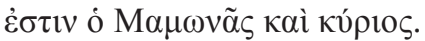

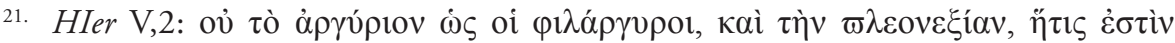

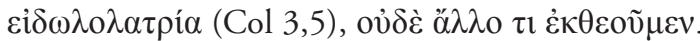

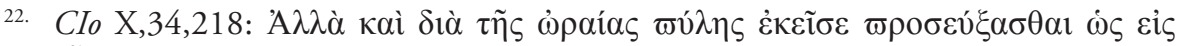

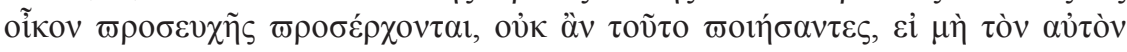

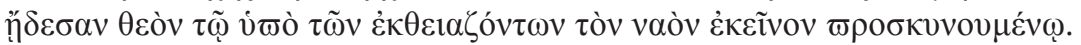




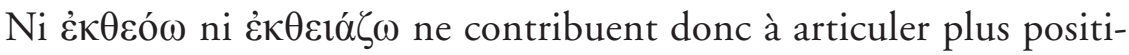
vement le discours de la déification, mais Origène n'éprouve aucun embarras à exploiter un verbe comme ảøo $\theta \varepsilon o ́ \omega$, plus directement lié aux expressions de la 'divinisation' dans le monde religieux gréco-romain, en particulier sous le terme $\alpha \dot{\sigma o} \theta \dot{\varepsilon} \omega \sigma t \varsigma$. Évidemment, il n’ignore pas sa signification péjorative, qu'il évoque une fois dans le Contre Celse ${ }^{23}$, mais il privilégie l'emploi positif du verbe, afin de décrire le processus de divinisation en relation à la conduite morale de l'individu. À ce propos nous constatons une convergence entre le 16ème Livre du Commentaire sur Matthieu et l'Homélie sur le Psaume 81, favorisée par la référence dans les deux cas à Ps 81,6-7. Si le sermon souligne le fait qu'en "faisant mourir les œuvres du corps" (Rm 8,13), nous "sommes déifiés" $(\dot{\alpha} \varpi \varepsilon \theta \varepsilon \dot{\theta} \theta \eta \mu \varepsilon v)^{24}$, le passage du Commentaire sur Matthieu insiste sur l'unique chance que Dieu donne à chacun par son appel à la béatitude. Cependant les hommes n'en veulent pas et choisissent au contraire de ne

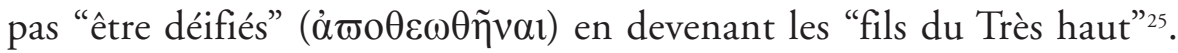

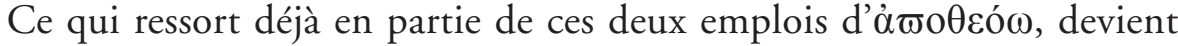
plus perceptible dans un fragment caténal sur Luc attribué à Origène: en effet, l'apothéose' envisagée se présente ici de manière encore plus explicite comme le résultat des efforts menés en vue de parvenir à la perfection spirituelle: à ceux donc qui "se sont déifiés", Dieu va se manifester "comme il est", "Dieu" pour des "dieux" 26.

Pour conclure ces brefs aperçus, il faut observer que ne sont pas attestés chez Origène les deux substantifs employés par les Pères pour indiquer la 'déification': ni $\theta \varepsilon ́ \omega \sigma ı \varsigma ~(o u ~ \theta \varepsilon i ́ \omega \sigma ı \varsigma)$, qui est de loin le mot le

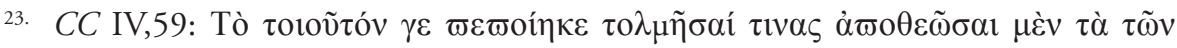

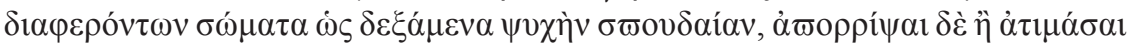

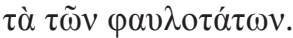

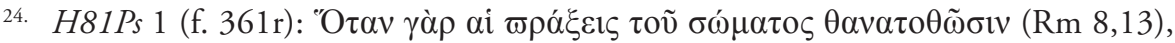

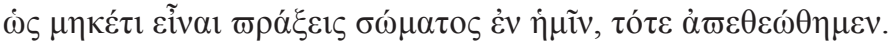

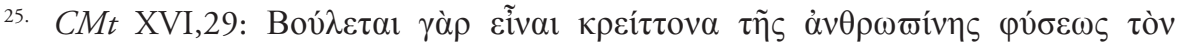

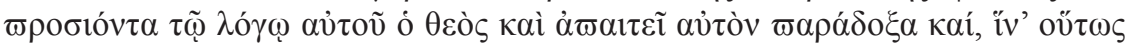

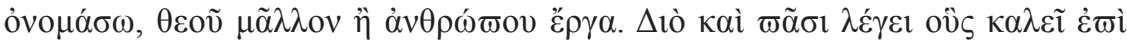

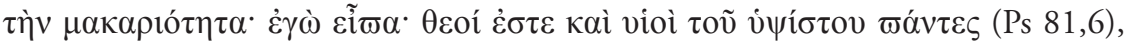

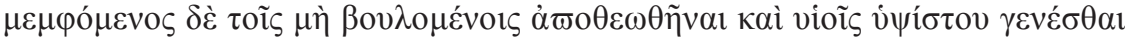

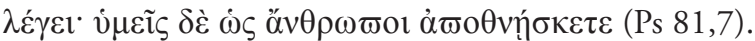

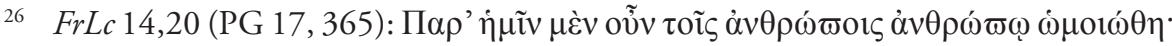

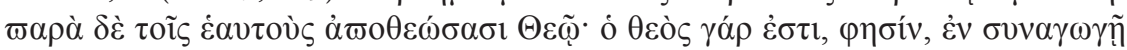
$\theta \varepsilon \tilde{\omega} \nu$ (Ps 81,1$)$. 


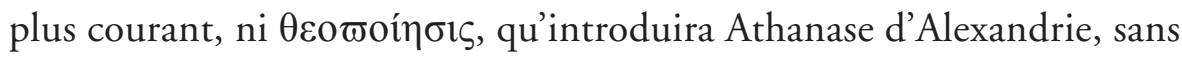
lui assurer par ailleurs une destinée durable à la différence du premier terme.

III. LE DOSSIER SCRIPTURAIRE À L'APPUI DE LA DÉIFICATION: ENJEUX EXÉGÉTIQUES ET DOCTRINAUX

La recherche terminologique que nous venons de mener reflète seulement en partie la richesse du discours origénien sur la déification. Il faudrait sans doute l'élargir à d'autres vocables, mais pour l'économie de cette contribution il est préférable de passer désormais à l'analyse du dossier scripturaire. Ceci nous permettra de pénétrer plus à fond dans l'élaboration de notre thème chez l'Alexandrin et d'en montrer la retombée plus vaste, car chaque articulation de sa pensée est tojours nourrie par l'aliment des Écritures. Or les passages ne manquent pas qui introduisent la perspective de la déification. Par le biais des occurrences scripturaires nous verrons alors se dessiner plus clairement l'agenda théologique' qu'Origène construit autour de notre thème. Même ici il faut par ailleurs sélectionner les lieux dans la masse de données. Souvent ils résultent du vis-à-vis de l'exégète avec le texte à commenter, mais en général ils laissent entrevoir une grille de références plus ou moins constantes. Autour de cette constellation prioritaire nous relevons la présence de citations plus occasionnelles, mais qui d'autre part témoignent la possibilité de nouveaux développements.

Parmi ces présences occasionnelles, nous observons l'emploi d'Ex 15,11 (“Qui est semblable à toi parmi les dieux, Seigneur?”), associé à Ps 81,1 et 6 dans la Géme Homélie sur l'Exode: puisque la Bible parle parfois de 'dieux' au pluriel et et les met en relation avec Dieu, Origène s'efforce, ici comme ailleurs, de préciser qu'il s'agit non des idoles ou des divinités du paganisme, mais bien de ceux que l'Écriture appelle 'dieux' (Ps 81,1 et 6), et qui sont tels "par grâce et participation" selon le texte latin de Rufin (per gratiam et participationem) ${ }^{27}$. Ce binome offre alors la clef pour expliquer, d'un côté, le fait que le texte sacré mentionne des

27. HEx VI,5: Quod dicit: quis similis tibi in diis (Ex 15,11), non simulacris gentium comparat Deum nec daemonibus, qui sibi falso deorum nomen adsciscunt, sed deus illos dicit, qui per gratiam et participationem Dei dii appellantur. De quibus et alibi Scriptura dicit: Ego dixi: dii estis (Ps 81,6), et iterum: Deus stetit in congregatione deorum (Ps 81,1). 
'dieux' et pour inculquer, de l'autre côté, la différence entre la divinité par grâce et la divinité par nature. Nous y dégageons à nouveau le thème de la 'ressemblance' a Dieu, qui - comme on l'a dit au début - reformule le thème de la déification en le situant dans la perspective de l' image de Dieu' dans l'homme. Dans ce cas, le renvoi à 1 Jn 3,2 ("Bien-aimés, dès maintenant, nous sommes enfants de Dieu, et ce que nous serons n'a pas encore été manifesté. Nous savons que lors de cette manifestation, nous lui serons semblables, parce que nous le verrons tel qu'il est") - un texte crucial du Nouveau Testament pour le motif de l'ó $\mu$ oí $\omega \sigma 1 \varsigma \theta \varepsilon \tilde{\omega}$, souvent évoqué à ce propos par l'Alexandrin - vient renforcer la distinction entre nature et grâce et conséquemment la différence des 'dieux' par rapport à $\mathrm{Dieu}^{28}$. Ainsi, la 'ressemblance' de l'homme avec lui, aboutissement final de son 'imitation de Dieu' - comme le rappelle entre autres le petit traité

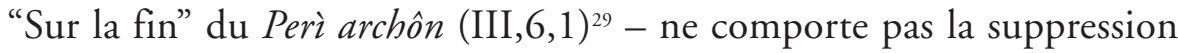
de la distinction de nature.

Nous pouvons rapprocher d'Ex 15,11, par exemple, les passages de Ps 76,14 ("Quel dieu est grand commen notre Dieu?") ou de Ps 95,5 ("Tous les dieux des nations sont des démons"). Il s'agit de textes semblables, car ils tracent également la différenciation par rapport aux faux dieux du paganisme, tandis qu'ils rappellent par contraste les 'dieux' dont il est permis de parler à juste titre dans les Écritures. Face à de tels textes, selon la 2ème Homélie sur le Psaume 76, il faut s'interroger sur les

28. HEx VI,5: Sed hi quamvis capaces sint Dei et hoc nomine donari per gratiam videantur, nullus tamen Deo similis invenitur vel in potentia vel in natura. Et licet Iohannes apostolus dicat: Filioli, nondum scimus quid futuri sumus; si autem revelatus nobis fuerit - de Domino scilicet dicens - similis illi erimus (1 Jn 3,2), similitudo tamen haec non ad naturam, sed ad gratiam revocatur. Comme l'observe Manlio Simonetti, "questo passo della lettera giovannea viene abitualmente interpretato da Origene per rilevare sia la somiglianza finale con Dio che sarà conseguita grazie ai meriti acquisiti sia la differenza tra l'attuale conoscenza di Dio e quella finale" (Origene. Omelie sull'Esodo, a cura di M. Simonetti, Roma, 2005, p. 180 n. 32). Il renvoie à Prin III,6,1; CMt XVII, 19; CRm V,9; VII,4.

29. Prin III,6,1: scilicet ut ipse sibi eam propriae industriae studiis ex dei imitatione conscisceret, quo possibilitate sibi perfectionis in initiis data per 'imaginis' dignitatem in fine demum per operum expletionem perfectam sibi ipse 'similitudinem' consummaret. Sed apertius haec et evidentius ita se habere Iohannes apostolus definit. (...) Per quod certissime indicat et finem omnium, quem adhuc sibi dicit ignotum, et similitudinem dei sperandam, quae pro meritorum perfectione praestabitur. Il faudrait aussi rappeler dans ce contexte le traité De promissionibus contenu dans Prin II,11 qui relie le desiderium Dei au thème de l'imago Dei dans l'homme. 
raisons de la comparaison entre Dieu et les 'dieux' ${ }^{30}$. Dans ce sermon Origène vient de citer les paroles de Jéthro en Ex 18,11 ("Je sais maintenant que le Seigneur est plus grand que tous les dieux"), en les critiquant expressément, à la suite de Philon d'Alexandrie (sans toutefois nommer celui-ci selon sa coutume plus habituelle), du fait que ces mots semblent vouloir comparer Dieu aux idoles ${ }^{31}$. Par ailleurs, étant donné qu'au dire de Paul "il y a, soit au ciel, soit sur la terre ceux qui sont dits dieux, comme il y a plusieurs dieux" (1 Cor 8,5), l'homéliste explique l'affirmation de l'Apôtre par la 'liberalité' de Dieu, qui répand ses bienfaits envers les êtres divinisés; en témoignent les deux passages de Ps 81,1 et 6, qui reviennent encore une fois pour compléter l'argumentation.

Ce texte de la 2ème Homélie sur le Psaume 76 - qui par ses propos anticipe de très près la teneur semblable de l'Homélie sur le Psaume 81 - nous présente de manière synthétique le principal dossier des textes scripturaires. À côté de l'emploi des versets 'canoniques' tirés précisément du Ps 81 , nous avons déjà noté celui de Ps 95,5, un passage exploité à plusieurs reprises par Origène dans son discours sur la déification, selon les deux registres désormais connus: en négatif et en positif ${ }^{32}$. Mais avec le texte de 1 Cor 8,5 (qui réplique lui aussi ces deux registres) et de 1 Cor 3,3 (qui dénonce pour sa part la conduite des hommes en tant qu'elle s'oppose à l'appel à la divinisation), nous repérons encore une allusion à Jn 10,34-35 ("N'est-il pas écrit dans votre Loi: 'J'ai dit: vous êtes des dieux??33. La loi appelle donc des dieux ceux à qui s'adressait la parole de

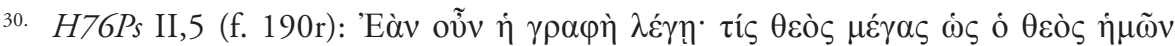

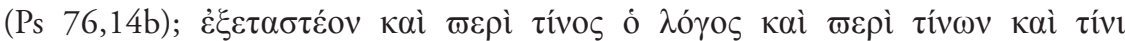

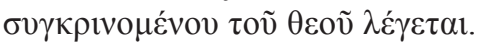

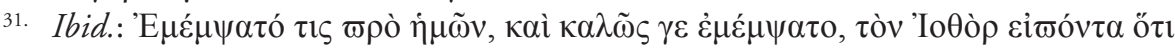

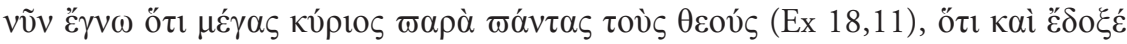

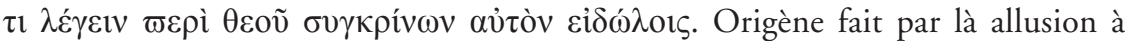
Philon, Ebr. 41-45.

32. Il n'est pas un cas que le recours à Ps 95,5 soit particulièrement fréquent dans le Contre Celse, à l'appui de la polémique menée contre le paganisme. Voir, par ex.,

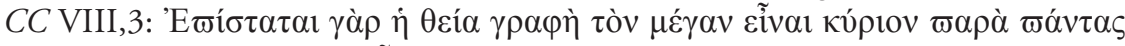

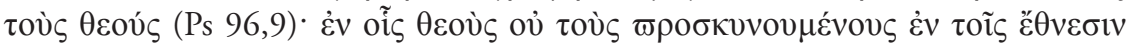

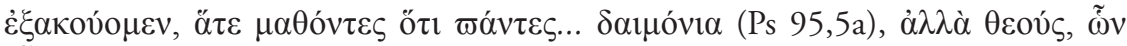

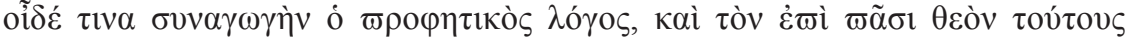

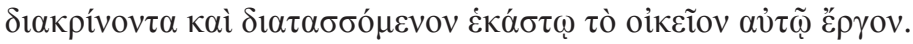

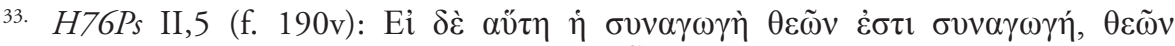

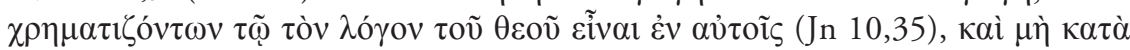

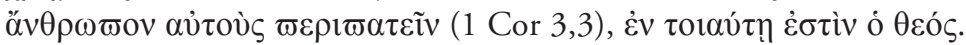


Dieu - et on ne peut abolir l'Écriture"). À cet égard, nous avons à faire à l'un des lieux scripturaires les plus importants pour l'Alexandrin quand il évoque la déification, notamment parce que celle-ci est sanctionnée par les paroles mêmes de Jésus, qui à leur tour reprennent le testimonium capital de Ps 81,6. En fait, le passage johannique indique plus directement l'agent responsable du processus qui rend les hommes des 'dieux' la venue du Verbe chez eux -, réaffirmant par là le rôle prédominant du Logos, que nous avons rencontré dans notre recherche terminologique (sans que mention soit faite du rôle du Saint-Esprit).

Dans le Prologue du Commentaire sur le Cantique, Origène cite expressément Jn 10,35 pour préciser les différentes façons d'utiliser le nom de 'dieu'. Il envisage alors un triple emploi de cette appellation: 1) principal, 2) impropre, 3) faux. Quoique l'on doive sans doute soupçonner une retouche de Rufin au texte grec original, en raison de l'énoncé trinitaire à la saveur trop fortement postnicéenne, la classification ainsi articulée respecte les données que nous avons dégagées jusqu’ici en retraçant le dossier scripturaire.

Comme par exemple le nom de Dieu aussi concerne principalement celui "de qui est tout, par qui est tout, en qui est tout" ( $\mathrm{Rm} \mathrm{11,36),}$ assurément parce qu'il exprime clairement la puissance et la nature de la triple unité. Mais en second lieu, et pour ainsi dire au sens impropre, l'Écriture appelle encore dieux "ceux à qui la Parole s'adresse" (Jn 10,35), comme le confirme le Sauveur dans les Évangiles. De plus, les puissances célestes semblent être appelées de ce nom quand il est dit: "Dieu se trouve dans l'assemblée des dieux, et au milieu il juge les dieux (Ps 81,1). Mais en troisième lieu, d'une manière non plus impropre mais fausse, les démons sont appelés dieux des nations, comme dit l'Écriture: "Tous les dieux des nations sont des démons" (Ps 95,5)

34. CCt Prol 2,34: Sicut, verbi gratia, et Deus nomen principaliter in eo est ex quo omnia et per quem omnia et in quo omnia $(\mathrm{Rm} 11,36)$, quod utique aperte virtutem et naturam trinae singularitatis enuntiat. Secundo vero in loco et, ut ita dixerim, abusive deos dicit Scriptura etiam illos ad quos sermo Dei fit (Jn 10,35), sicut confirmat Salvator in evangeliis. Sed et caelestes virtutes sub hoc nomine appellari videntur, cum dicitur: Deus stetit in congregatione deorum, in medio autem deos discernit (Ps 81,1). Tertio vero in loco non iam abusive, sed falso dii gentium daemones appellantur, cum dicit Scriptura: Omnes dii gentium daemonia (Ps 95,5). L'inflexion au sens trinitaire de $\mathrm{Rm}$ 11,36 est confirmée si l'on compare l'exégèse donnée sur ce passage en $C R m$ VIII, 13 avec CC VI,65. 
Cette tripartition, en reconnaissant la légitimité de l'emploi 'im-

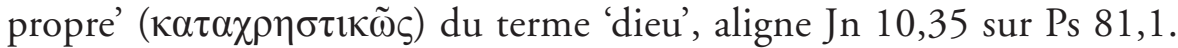
Ces deux testimonia s'attirent, pour ainsi dire, l'un l'autre, comme nous le constatons aussi dans l'Homélie sur le Psaume 81, lorsqu'il s'agit de commenter le v. 6 ("J'ai dit: Vous êtes des dieux et des fils du Très Haut, vous tous") ${ }^{35}$. Au même temps, après l'énoncé général sur ceux qui peuvent être appelés 'au sens impropre' des 'dieux', le texte du Commentaire sur le Cantique focalise cette appellation sur les anges. Comme nous le montrerons par la suite, il s'agit d'une identification supportée plus spécifiquement par l'exégèse du Ps 81 , sans que celle-ci réduise d'autre part la divinisation aux seules puissances célestes.

En effet, nous arrêtant encore un instant sur Jn 10,35, il faut souligner que le passage johannique amène Origène à identifier d'autres figures de 'dieux', comme les prophètes et les apôtres. Selon le 2ème Livre du Commentaire sur l'Épître aux Romains, si le Seigneur nomme 'dieux' ceux auxquels le Verbe de Dieu s'adresse, on doit admettre que David et Paul, étant justement dans la condition de destinataires de la Parole divine, ne sont pas des hommes mais bien des 'dieux' ${ }^{36}$. Par là, David et Paul s'ajoutent aux trois patriarches Abraham, Isaac et Jacob, qui selon la 2ème Homélie sur le Psaume 76, rappelée plus tôt, ont titre eux aussi à être regardés comme des 'dieux' ${ }^{37}$.

À la différence de Jn 10,35, l'Alexandrin ne semble pas trop exploiter un autre lieu néotestamentaire qui présente l'idée de la déification en des termes assez éloquents: 2 P 1,4 (“... les plus grandes promesses nous

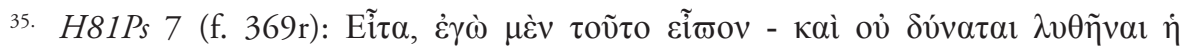

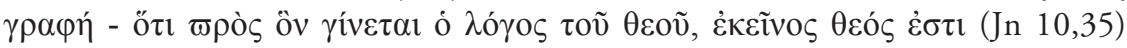

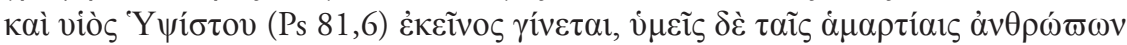

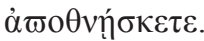

36. CRm II,14: Sed si redeamus ad interiorem scripturae intellectum, inveniemus, quod omnes prophetae vel apostoli ex illis sint, ad quos sermo Dei fit, sicut scriptum est: Et factum est verbum Domini ad illum vel illum prophetam. Hos ergo, ad quos sermo Dei fit, Dominus in evangelio non homines pronuntiat esse, sed deos. Sic enim dicit, quod si illos dicit deos, ad quos sermo Dei fit, et non potest solvi Scriptura (Jn 10,35). Quia ergo et ad David tamquam prophetam et ad Paulum tamquam apostolum sermo Dei factus est, sine dubio non erant homines, sed dii, ad quos sermo Dei factus est.

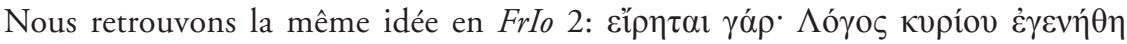

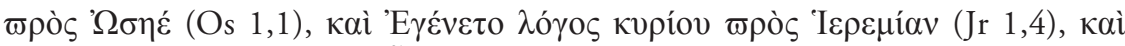

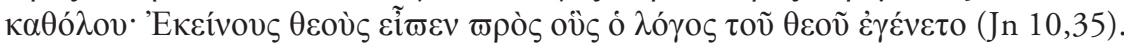

37. Voir supra n. 17. 
ont été données, afin que vous deveniez ainsi participants de la divine nature, vous étant arrachés à la corruption qui est dans le monde") ${ }^{38}$. Si la 4ème Homélie sur le Lévitique recourt à ce passage, elle l'utilise pour

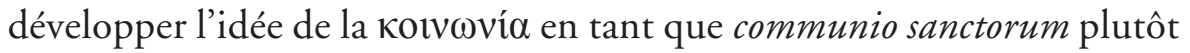
que pour y répérer expressément des arguments au profit de la déification $^{39}$. Au contraire, l'emploi par le 4ème Livre du Commentaire sur l'Épître aux Romains s'avère être plus riche ${ }^{40}$. Ici la participation à la nature divine découle de la source trinitaire de Dieu en tant que caritas: cet amour se répand, à partir de sa source primordiale dans le Père, dans le Fils et le Saint-Esprit, qui sont tous les deux 'amour' à leur tour; grâce à eux les "coeurs des saints parviennent à participer de la nature divine" ${ }^{41}$.

Nous avons déjà mentionné la citation de 1 Cor 8,5, parfois reprise par Origène en liaison avec le v. 6 ("Car bien qu'il y ait, soit au ciel, soit sur la terre, de prétendus dieux, - et de fait il y a quantité de dieux et quantité de seigneurs, - pour nous en tout cas, il n'y a qu'un seul Dieu, le Père, de qui tout vient et pour qui nous sommes faits, et un seul Seigneur, Jésus Christ, par qui tout existe et par qui nous sommes"). L'Alexandrin exploite positivement l'ambivalence du passage paulinien en vue de soutenir la déification, comme nous le voyons dans la lère Homélie sur le Psaume 15, où il explique les 'nombreux dieux' de l'Apôtre

38. Selon l'avis de Martens, «Divinization", cit., 91: "He [scil. Origen] does not make much (etymologically) of the occurrence of the idea in 2 Peter 1:4, although the ideas represented there are the substrate of all his anthropology and cosmology".

39. $H L v$ IV,4.

40. CRm IV,9,15: Quod si et spiritus caritatis et filius caritatis et Deus caritas invenitur, certum est quod ex uno paterno deitatis fonte et filius intellegendus est et Spiritus Sanctus ex cuius abundantia etiam sanctorum cordibus ad participationem capiendam divinae naturae sicut Petrus apostolus edocuit (2 P 1,4) abundantia caritatis infunditur, ut per istud Sancti Spiritus donum compleatur ille sermo quem Dominus dicit: Sicut tu pater in me et ego in te et isti in nobis unum sint divinae scilicet naturae participes effecti in abundantia caritatis per Sanctum Spiritum ministratae.

41. Comme l'observe Francesca Cocchini, ce 'modèle triangulaire' de la Trinité est moins commun chez Origène que le 'modèle vertical' ou hiérarchique (Origene, Commento alla Lettera ai Romani. Libri I-VII, a cura di F. Cocchini, Casale Monferrato, 1985, p. 223 n. 40). En fait, les parallèles avec le Commentaire sur le Cantique justement signalés par Michel Fédou (Origène, Commentaire sur l'Épître aux Romains. Livres III-V, SC 539, Paris 2010, p. 316 n. 1) nous livrent seulement la référence au Père et au Fils. Cf. CCt Prol 2,26; II,4,16; III,10,7. 
par la référence à Ps $81,6^{42}$. Au même temps ce testimonium prélude indirectement à une 'hiérarchisation' des êtres divinisés, qui revient en forme plus directe dans le 1er Livre du Commentaire sur Jean. Ici Origène s'appuyant d'abord sur Ps 135,2 ("Confessez le Dieu des dieux") et Ps 49,1 ("Le Seigneur, Dieu des dieux, a parlé") et rappelant à leur suite l'emploi paulinien du terme 'dieux' selon l'usage 'prophétique' - c'està-dire dans les textes des Psaumes - exploite 1 Cor 8,5 pour introduire l'échelle des êtres spirituels, des anges jusqu'à l'homme, dans la ligne de l'exposé sur les 'natures rationnelles' du Perì archôn (Prin I,5,1) ${ }^{43}$. Il rappelle encore le même passage dans le 4ème Livre du Contre Celse, le faisant précéder comme sa clef interprétative par la citation de Ps 81,1 et Ps 95,5, qui - comme on l'a vu - tracent respectivement la ligne de distinction entre les vrais et les faux 'dieux'. Ceux-ci sont par ailleurs à identifier surtout avec les anges. Mais dans le 8ème Livre, Origène plaide pour "un discours plus profond et secret concernant les dieux et

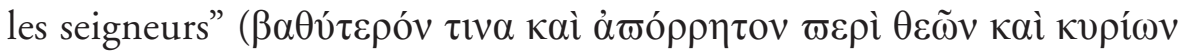
$\lambda$ ó $\gamma$ ov), avec l'appui d'un dossier scripturaire qui rassemble à nouveau plusieurs des lieux que nous venons de mentionner (Ps 96,9; Ps 95,5; Ps 81,1 ; Ps 49,1; Ps 135,2-3, ainsi que 1 Cor 8,5) ${ }^{44}$. En cet endroit, non seulement il fait allusion au discours sur les natures rationnelles, mais il a le souci de distinguer le rôle éminent du Christ en tant que 'Dieu' et 'Seigneur' ${ }^{4}$. Finalement, un fragment du Commentaire sur Isaïe, trans-

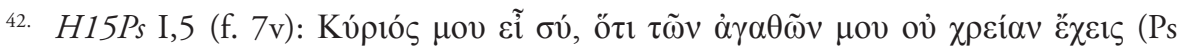

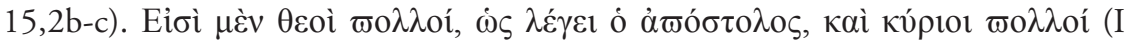

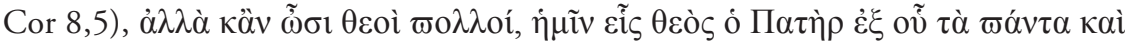

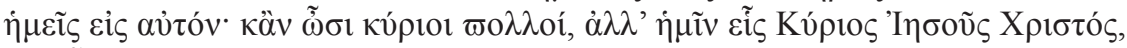

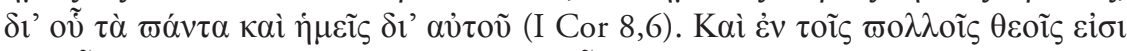

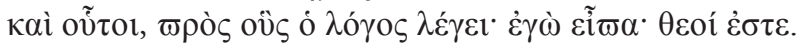

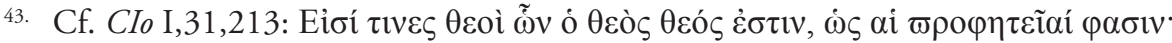

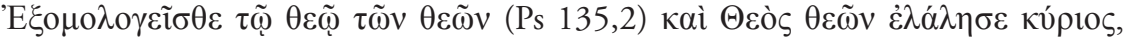

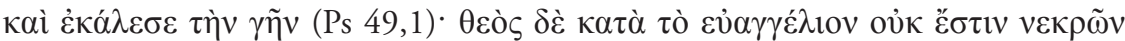

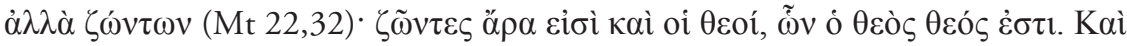

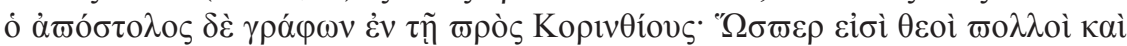

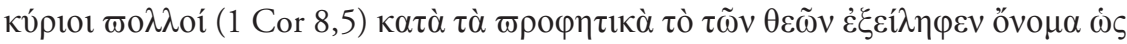
$\tau v \gamma \chi \alpha v o ́ v \tau \omega v$.

44. CC VIII,3-4.

45. Nous constatons le recours à 1 Cor 8,5 dans un autre texte qui revient peut-être

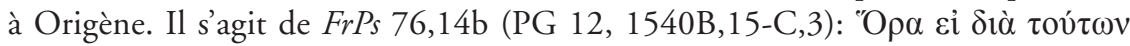

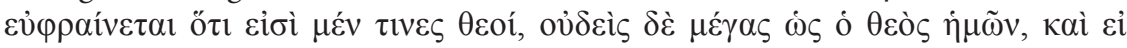

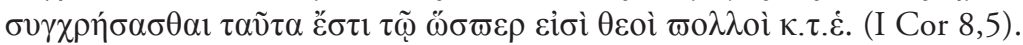


mis dans l'Apologie d'Origène écrite par Pamphile, tout en maintenant la vision hiérarchique souvent tirée de 1 Cor 8,5-6, élargit son application au sens trinitaire par une triple reconnaissance de l'action de l'Esprit, du Christ et du Père sur les êtres; cela revient à dire que, selon un ordre progressif, il y a des 'pneumatiques', des 'christs' et des 'dieux'46.

Pour compléter cette présentation du dossier scripturaire sur la déification, il faudrait encore se souvenir du fait que celle-ci, en tant qu'elle réalise l'assimilation de l'homme à l'image du Fils, comporte également, pour ainsi dire, une 'christification': le saint ou le parfait devient alors un alter Christus. À ce propos, Origène recourt très souvent à 2 Cor 13,3 ("Vous voulez, n'est-ce pas, une preuve que le Christ parle en moi"). Si l'identification au Christ représente une urgence qui pousse le prédicateur à invoquer son assistance - comme nous le voyons dans la 2ème Homélie sur le Psaume 15 ainsi que dans d'autres sermons ${ }^{47}$-, dans la lère Homélie sur la Genèse le texte de 2 Cor 13,3 contribue à orchestrer l'idée de la demeure de Dieu chez les saints, ce qui à son tour refaçonne le motif de la 'déification' en la situant sous un nouveau jour, précisément par l'idée de l'inhabitation divine dans l'homme, specialement dans son 'cœur'48. Selon la 3ème Homélie sur le Psaume 76, celui qui devient "homme de Dieu authentique ( $\gamma v \eta ́ \sigma t o \varsigma)$ ", peut grâce au "Christ qui parle en lui" commander les éléments de la nature au même titre que Josué 49 . Pour ce sermon, l'homme de Dieu' arrive à disposer de pareils pouvoirs du fait qu'étant une "race digne d'honneur" (Sir 10,19) il ac-

46. CIs I = Apol 116: Sicut enim cum unus sit Spiritus sanctus tanti per singulos dicuntur spiritus sancti quanti sunt hi qui habent in se Spiritum sanctum, ita et de Christo dicendum est; ab uno enim Christo multi christi fiunt de quibus dicit Scriptura: Nolite tangere christos meos et in prophetis meis nolite malignari (Ps 104,15); sic et ab uno Deo multi dii dicuntur, omnes scilicet hi in quibus habitat Deus, sed nobis unus est Deus Pater ex quo omnia (I Cor 8,6).

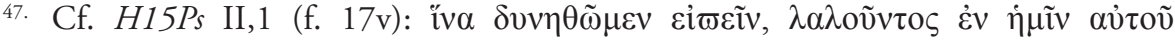

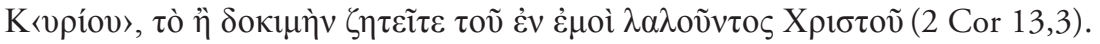

48. HGn I,13: Et non solum requiescit super eos Deus, sed et inhabitat in iis. Si vero tantus quis effici potest, ut possit dicere: aut documentum quaeritis eius, qui in me loquitur Christus? (2 Cor 13,3), in hoc non solum inhabitat Deus, sed etiam inambulat.

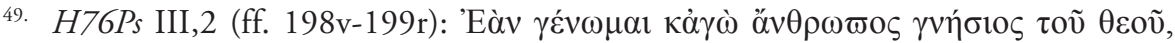

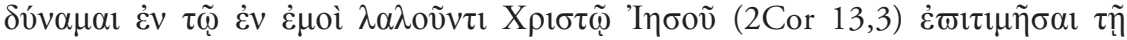

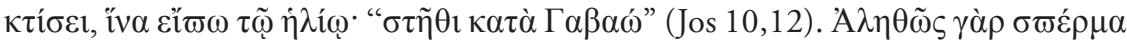

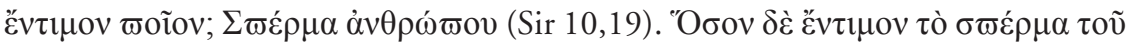

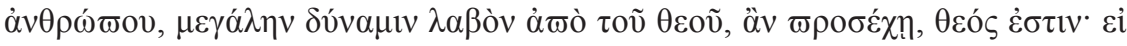

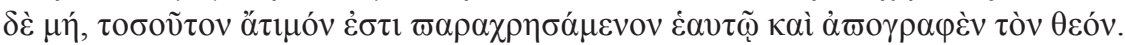


complit la vocation de sa 'race' et par là devient dieu. La 2ème Homélie sur le Psaume 80, en commentant le v. 10 ("Qu'il n'y ait point chez toi un dieu d'emprunt") reprend le même point de vue et oppose la condition du 'saint', dans lequel le Christ parle, à celle du 'pécheur' habité par les démons des vices ${ }^{50}$. Le Gème Livre du Commentaire sur Jean résume de quelque sorte ces motifs juxtaposant en pratique l'idée de déification à celles de 'christification', de 'participation' ou d'inhabitation': le Christ apparaît ici comme la 'vie' des fidèles et c'est en vertu de l'imitation du Christ et de la conformation à son image que les fidèles deviennent d'autres $\chi \rho ı \sigma \tau o i ́$, suivant celui qui est à l'image de Dieu ${ }^{51}$.

En passant en revue les dossiers scripturaires rassemblés par Origène, nous avons opéré une sélection par nécessité, étant donné le vaste rayonnement biblique de notre thème mis en évidence par l'Alexandrin, à travers l'Ancien et le Nouveau Testament. Cependant, nous avons pu élargir le spectre thématique et conceptuel portant sur la déification, grâce à des testimonia scripturaires qui parviennent à la dessiner dans une perspective plus riche et complexe et la résument, en particulier, dans le motif de la 'conformation au Christ'. Celui-ci par ailleurs nous fait souvenir du thème de la filialité divine de l'homme et de l'inhabitation de Dieu en lui comme son 'temple'. L'Alexandrin en parle beaucoup dans ses œuvres, spécialement dans le commentaire du Notre Père faisant partie du Traité sur la prière. Il exploite à cet égard, en plus du motif de Dieu se promenant dans le paradis terrestre (Gn 3,8), un texte paulinien - 2 Cor 6,16 - qui reprend Lv 26,12 ("Or, c'est nous qui le sommes, le temple du Dieu vivant, ainsi que Dieu l'a dit: 'J'habiterai au milieu d'eux et j'y marcherai; je serai leur Dieu et ils seront mon

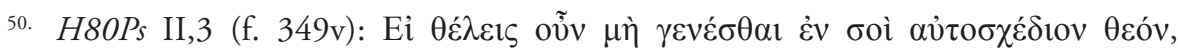

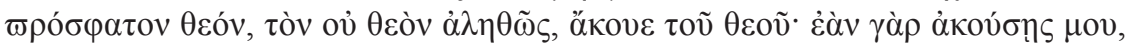

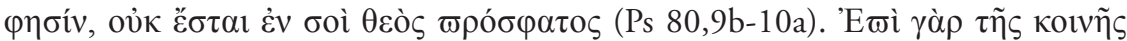

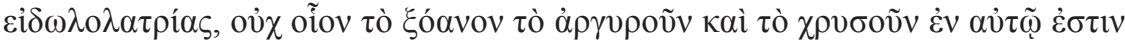

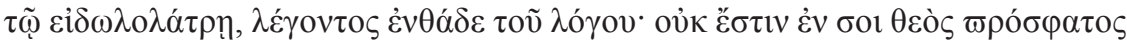

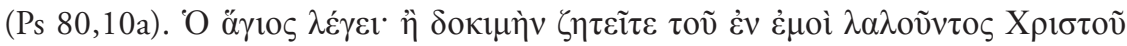
(2 Cor 13,3); 'O $\delta \dot{\varepsilon} \alpha \dot{\alpha} \mu \alpha \rho \tau \omega \lambda$ ò $\varepsilon^{1}$

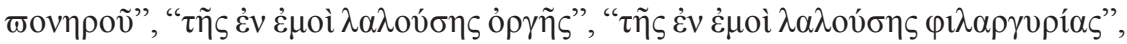

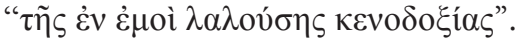

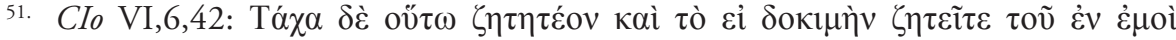

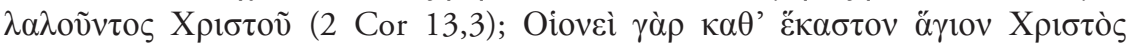

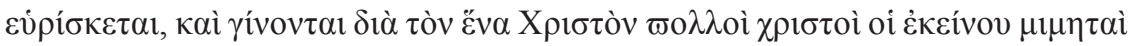

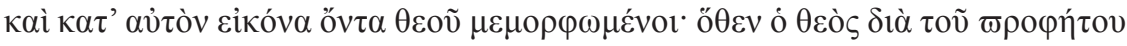

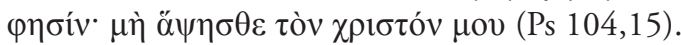


peuple"'): de cette manière, le Père avec le Christ se promènent au sein de celui qui est devenu parfait ou saint comme dans un 'paradis spirituel's2. Le Contre Celse, faisant appel lui aussi à 2 Cor 6,16 y ajoute l'intervention de l'Esprit du Christ et souligne l'acquisition de la condition de 'temple' spirituel moyennant l'imitation de Dieu's3. Néanmoins, ce texte insiste également lui aussi sur le fait que le Père et le Fils établissent leur demeure dans le fidèle, d'autant plus qu'il se sert de Jn 14,23 ("et nous viendrons à lui et nous ferons chez lui notre demeure").

Il est évident qu'il faudrait verser aussi ce testimonium dans le dossier déjà si abondant des passages scripturaires à l'appui du discours origénien sur la déification. L'Alexandrin s'en est servi à plusieurs reprises dans le Traité sur la prière et ailleurs, souvent en combinaison avec Ap 3,20 ("Voici que je me tiens à la porte et je frappe; si quelqu'un entend ma voix et ouvre la porte, j'entrerai chez lui pour souper, moi près de lui et lui près de moi"), un texte-clef pour sa doctrine de la nourriture spirituelle et comme tel faisant également partie du discours sur la

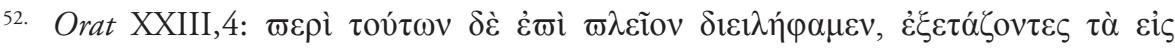

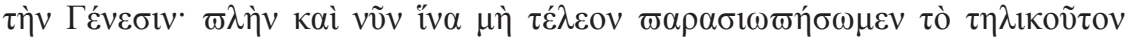

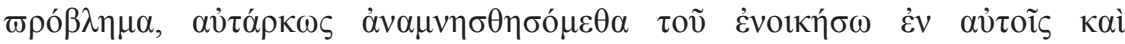

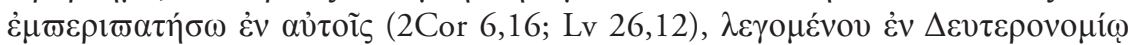

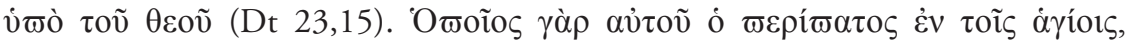

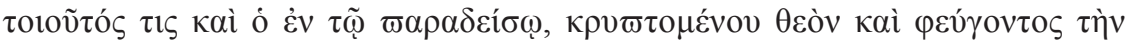

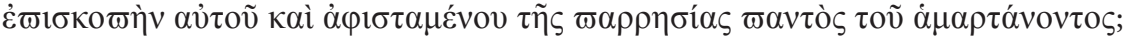

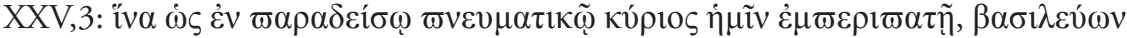

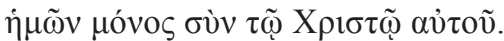

53. Cf. CC VIII,18. Le thème du temple et de l'inhabitation de Dieu est témoigné par

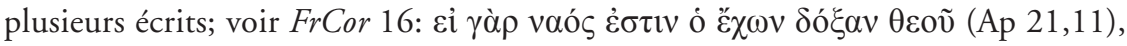

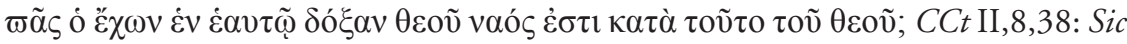
enim dicit hic rex quia: habitabo in is et inambulabo in iis (Lv 26,12), profecto qui tantam Verbo Dei cordis sui latitudinem praebent, ut etiam in iis deambulare dicatur, spatiis scilicet amplioris intelligentiae agnitionisque diffusioris; CRm II,5: Idcirco ergo Deus de sanctis suis quos dilatari novit et habere intra cordis sui domicilium spatia longe lateque diffusa dicit: habitabo... (2Cor 6,16); HGn I,13 (supra, n. 48); HEx VIII,4: Si Dei sumus, tales esse debemus, ut compleatur illud, quod dicit de nobis Deus: quia habitabo... populus (2 Cor 6,16); HLv VII,2: Vult enim in isto corpore ecclesiae suae et in istis membris populi sui ipse velut anima habitare, ut omnes motus atque omnia opera secundum ipsius habeat voluntatem, ut vere compleatur in nobis illud prophetae dictum: habitabo... inambulabo (Lv 26,12). Voir, à ce propos, C.L. Rossetti, 'Sei diventato Tempio di Dio'. Il mistero del Tempio e dell'abitazione divina negli scritti di Origene, Roma, 1998. 
déification ${ }^{54}$. Cette dernière référence ne figure pas dans les homélies du codex de Munich, mais le thème des aliments spirituels y occupe une place importante (spécialement dans les Homélies sur le Psaume 77), de même que le motif du 'lieu de Dieu'. Ainsi, la 2ème Homélie sur le Psaume 67 l'introduit à propos du v. 5b ("préparez une voie à celui qui avance vers occident"): le Seigneur recherche non seulement une 'voie' (óoóv) chez le fidèle mais encore plus une 'maison' (oî́kov) pour demeurer et se promener en lui ${ }^{55}$. De la même manière l'Homélie sur le Psaume 75 développe l'exégèse du v. 3 ("et son lieu fut en paix") affirmant que le saint - appelé ici classiquement comme le $\kappa \alpha \lambda$ ò $\varsigma \alpha \alpha \grave{~} \alpha \gamma \alpha \theta o ́ s-$ ne cesse de se fatiguer pour procurer en soi "un lieu pour le Seigneur" et "donner une habitation" au Christ, selon la promesse divine de Lv 26,12. Elle exemplifie par ailleurs la réalisation de cette promesse dans le prohète Isaïe, car Dieu parlait à travers lui ainsi qu'à travers les autres prophètes. Nous rejoignons par là le motif de la venue du Logos dans l'âme de ceux qui sont déifiés, comme nous l'avons constaté précédemment ${ }^{56}$.

\section{Un TESTIMONIUM CAPITAL: L'EXÉGÈSE DU PSAUME 81}

Notre inventaire a mis en lumière le relief tout à fait unique qu'assume le Psaume 81, avec ses deux versets 1 et 6 . Il s'agit du testimonium

54. Cf. Orat XXVII,11 et les autres passages que j'ai rassemblés dans mon livre $\mathrm{La}$ preghiera secondo Origene. L'impossibilità donata, Brescia, 2011, p. 226 n. 684.

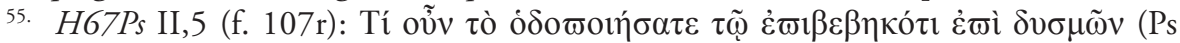

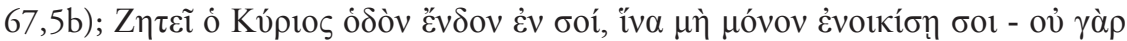

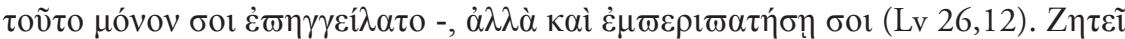

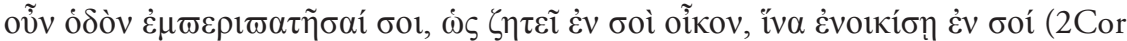
$6,16)$.

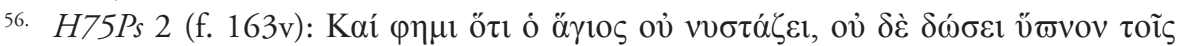

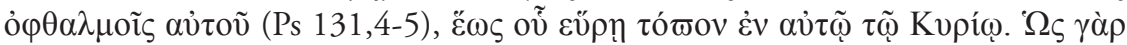

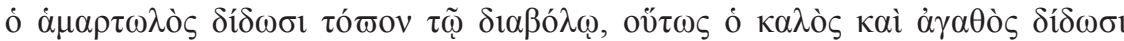

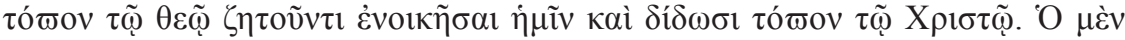

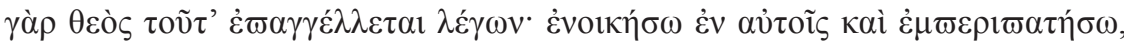

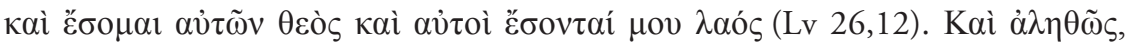

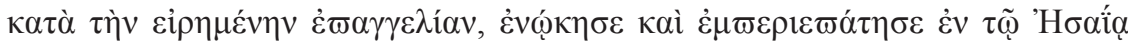

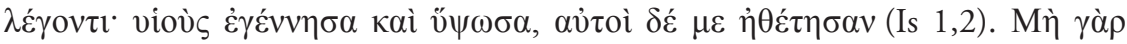

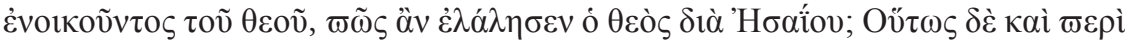

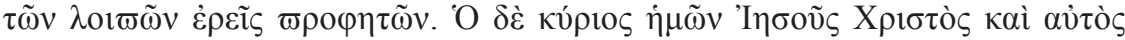

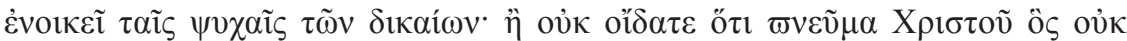

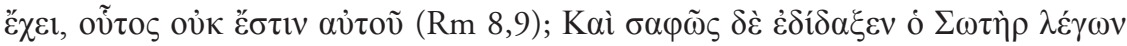

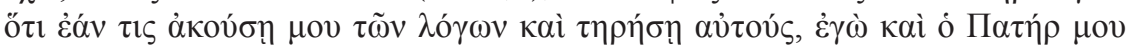

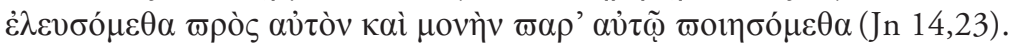


scripturaire par excellence pour la doctrine patristique de la déification. En tant que tel il revient très fréquemment chez Origène, soit dans les Homélies sur les Psaumes soit dans ses autres écrits, mais nous avons maintenant la chance de parcourir en entier l'exégèse que l'Alexandrin a donnée de ce psaume grâce à la dernière homélie dans la collection du Codex de Munich. L'analyse de ce sermon s'avère être tout à fait intéressante, puisqu'Origène, à côté des motifs plus habituels et notoires, qui soutiennent normalement sa réflexion sur la déification, ajoute un approfondissement peu usuel concernant l'objet' de la déification. Il y fait valoir comment ce procès investit l'homme en sa totalité: non seulement l'esprit et l'âme, mais aussi le corps. L'accent placé sur le corps 'déifié' est quelque peu surprenant, mais - comme nous le verrons par la suite - il est pleinement compatible avec l'approche origénienne de la doctrine de la résurrection de la chair, à partir de la réflexion sur le corps ressuscité du Christ.

Pour son interprétation du Psaume 81, l'Alexandrin ne semble pas dépendre directement de la tradition exégétique antécédente, quoiqu'il soit raisonnable de supposer qu'il la connaissait. Avant lui nous observons, en effet, une valorisation de l'apport de ce psaume en vue d'argumenter la déification chez Justin, Irénée de Lyon et Clément d'Alexandrie, c'està-dire des auteurs (surtout les deux derniers) qu'Origène ne devait pas ignorer. D'autre part, il n'est pas exclu qu'il ait connu des éléments de la tradition exégétique juive, comme le laisse soupçonner l'application particulière de Ps 81,1 (en combinaison avec Ex 22,27) dans l'exposé du Contre Celse sur la 'constitution des Juifs' (CC IV,31): Origène y fait allusion à propos des juges honnêtes, qui selon "une coutume nationale"

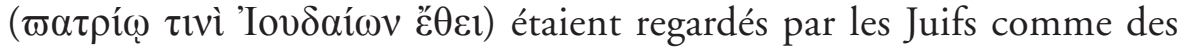
'dieux' ${ }^{57}$. Une application de ce type reste cependant isolée par rapport à l'emploi plus commun chez l'Alexandrin, qui interprète les 'dieux' de

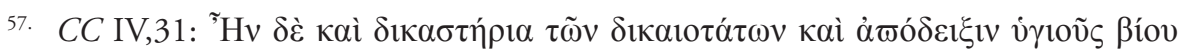

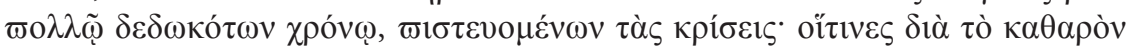

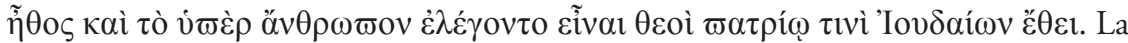
mention d'une "coutume nationale" laisse penser à des traditions exégétiques juives, ce qui serait conforme à l'empreinte laissée par celles-ci dans l'exposé apologétique, à côté des révisions de la Septante. Cf., à ce propos, mon article "Die "Verfassung der Juden": Das biblische Judentum als politisches Modell in Origenes' Contra Celsum», Zeitschrift für antikes Christentum, 7, 2003, p. 310-328, spécialement les pp. 324-325 et les notes 63-64. 
Ps 81,1 et 6 comme les anges, les prophètes, les justes ou les saints. En tout cas, ces précédents exégétiques, soit chrétiens soit juifs, avaient déjà ébauché en grande partie l'idée sotériologique, selon laquelle ce psaume renferme la vocation de l'homme à la filialité avec Dieu ${ }^{58}$.

Dans l'Homélie sur le Psaume 81 cet horizon sotériologique se manifeste dès le début, par le petit prologue qui introduit le commentaire suivi du texte en le plaçant sous le signe du progrès spirituel. Il exploite à cette fin les paroles de Mt 10,25 ("Il suffit que le disciple devienne comme son maître, et le serviteur comme son patron"), pour inculquer l'idée que le Maître est venu sur terre afin de transformer ses disciples et les rendre semblables à lui. Or ce Maître est non seulement le Christ, qui veut que nous devenions à notre tour des $\chi \rho 1 \sigma \tau o$, mais il est aussi un Dieu, qui nous appelle à le devenir également. Nous retrouvons donc en ouverture l'idée de la conformation au Christ, en tout ce qu'il représente, car tous ses 'attributs' sont à partager par les disciples. Par conséquent, nous entrevoyons - peut-être plus ouvertement qu'ailleurs - l'impact de la doctrine origénienne des ėøívorar du Christ sur le discours de la déification autant que sa reformulation en lien avec l'idée paulinienne de la conformation au Christ $^{59}$.

58. Sur les débuts de l'exégèse patristique et ses antécédents juifs, voir C. Mosser, «The Earliest Patristic Interpretations of Psalm 82. Jewish Antecedents, and the Origin of Christian Deification", Journal of Theological Studies, 56, 2005, p. 3074. Il s'agit certainement d'une contribution importante, mais à mon avis trop univoque: elle ne s'interroge pas sur l'application de Ps 81,1 et 6(-7) au-delà de sa ligne d'interprétation, qui revient à considérer ces versets comme un condensé de l'histoire du salut. Pour d'autres approches voir A. van den Hoek, "I Said, You are Gods...”. The Significance of Psalm 82 for Some Early Christian Authors", in The Use of Sacred Books in the Ancient World, Leuven, 1998, 203-219; M.D. Nispel, "Christian Deification and the Early Testimonia», Vigiliae Christianae, 53, 1999, p. 289-304.

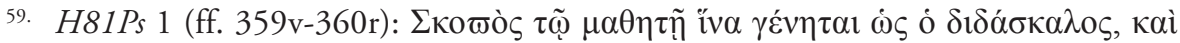

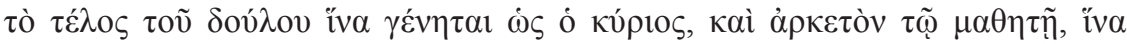

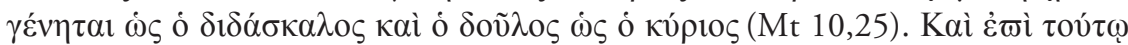

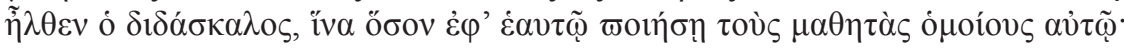

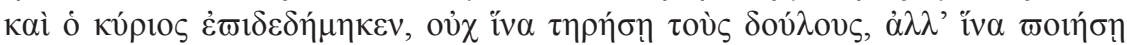

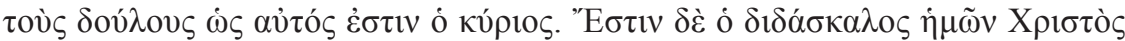

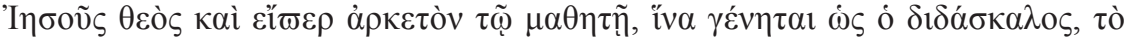

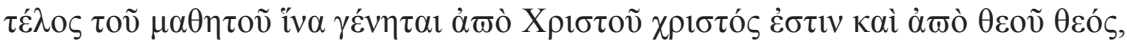

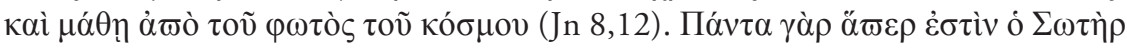

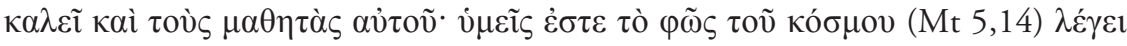

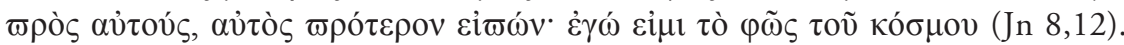




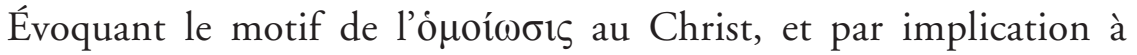
Dieu, notre homélie rejoint l'idée de l'imitation de Dieu' se donnant à la suite de l'imitation du Christ', que nous retrouvons dans la 2ème Homélie sur le Psaume 15, où le prédicateur fait appel à 1 Cor 11,1 ("Devenez mes imitateurs, comme je le suis moi-même du Christ"). Origène se demande alors si l'homme doit se faire imitateur du Premier-Né de toute créature (Col 1,15), et par là de la Sagesse, du Logos, de la Vérité, ou s'il peut l'être seulement de l'homme Jésus. Il y répond par un renvoi au dynamisme du progrès spirituel, soulignant que celui qui avance sur le chemin de la perfection est en mesure d'imiter non seulement la "divinité du Christ", mais même la "divinité du Dieu de l'univers"60, le fondement scripturaire étant assuré par les appels divins à la perfection et à la sainteté contenus dans l'Ancien et le Nouveau Testament $(\mathrm{Mt}$ 5,48; Lv 11,45; Dt 18,13). De son côté, la 2ème Homélie sur le Psaume 67 esquisse, sans montrer maintenant aucune hésitation, le portrait du "juste en tant qu'imitateur de Dieu”: du fait qu'il est devenu un 'fils de

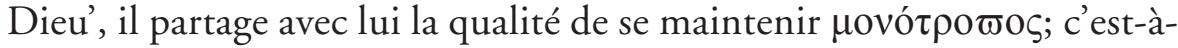
dire, il est capable de rester toujours le même, sans subir des altérations dans sa conduite vertueuse ${ }^{61}$. En effet, comme le souligne pour sa part le Contre Celse, la vertu est la même, du point de vue qualitatif, en Dieu et en l'homme; par conséquent, la pratique des vertus permet à l'homme d'assumer sur lui-même les "caractères de Dieu" ${ }^{2}$.

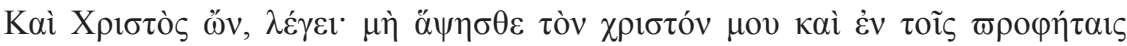

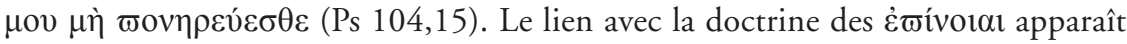
aussi dans l'emploi que CIo XXXII,10,117 fait de Mt 10,25, mais sans porter expressément sur la déification.

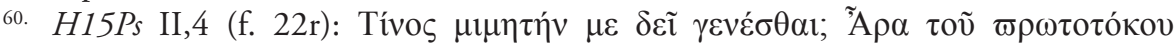

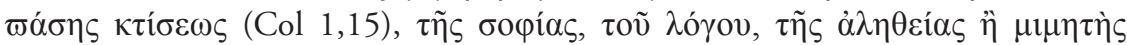

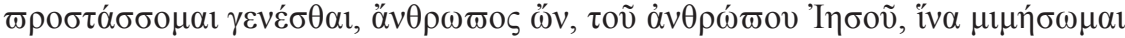

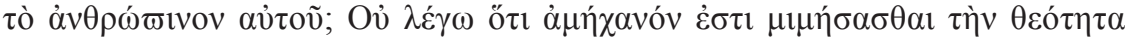

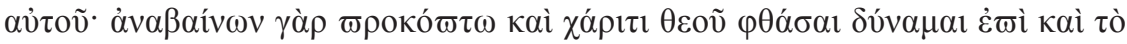

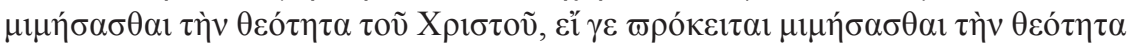

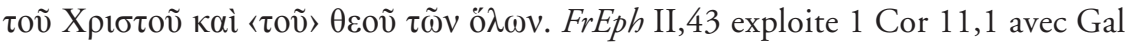
2,20 et Eph 4,19 uniquement pour l'idée de la conformation au Christ.

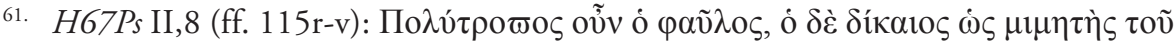

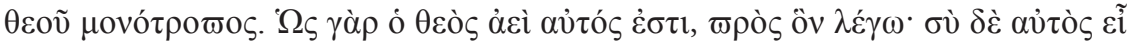

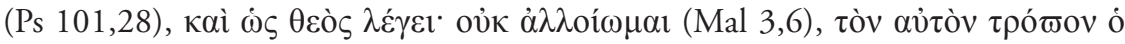

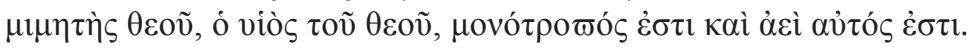

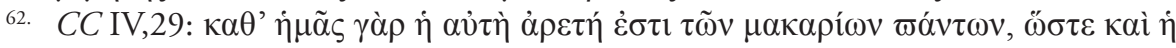

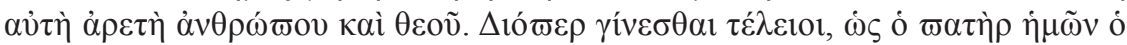


Dans l'Homélie sur le Psaume 81 le motif de l'imitation de Dieu reviendra par la suite sous forme d'exhortation, au moment où le prédicateur incite ses auditeurs à se faire des imitateurs de Dieu et devenir ainsi ses enfants ${ }^{63}$. La note morale et spirituelle, sans doute dictée, avec son urgence pratique, par la situation homilétique (qui aboutira ensuite à un appel à la sainteté universelle des fidèles), inspire en premier lieu le motif de la déification, comme nous le constatons par l'exégèse du v. 1. Origène y applique l'image scripturaire de l'“assemblée des dieux", au milieu de laquelle Dieu siège pour juger, à la communauté ecclésiale, pourvu que celle-ci abandonne les "œuvres de la chair" (Gal 5,19) et produise les "fruits de l'Esprit" (Gal 5,22). Dans ce dernier cas, notre conduite n'est plus celle des hommes, mais elle est propre de 'dieux', car nous sommes simplement des 'hommes', lorsque nous "tombons de la divinité". Une telle 'chute' laisse deviner au premier abord une allusion à la doctrine de la préexistence (surtout par la référence au v. 7 , avec l'image de l'“un des princes qui est tombé"), mais s'il est sans doute permis de la supposer, il faut reconnaître qu'elle reste bien cachée dans la suite de l'exposé, ou même qu'elle ne s'avère pas indispensable aux fins de l'argumentation. En fait, l'Alexandrin explique cette 'chute' par la référence à 1 Cor 3,3-4, en denonçant la conduite "bien humaine" de la communauté de Corinthe avec toutes ses dissensions et disputes, tandis qu'il reformule les propos de l'Apôtre en ce sens: "Le Logos vous appelle à devenir des dieux, mais vous vous conduisez comme des hommes". La 'chute', alors, est plutôt celle qui dérive de l'agir de l'homme, non conforme au 'don' ( $\delta \omega \rho \varepsilon \alpha ́)$ de la 'divinité' que Dieu lui octroie ${ }^{64}$. Selon un schéma, qui dispose d'un vaste soutien dans la pensée d'Origène,

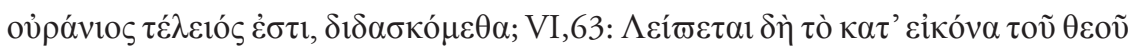

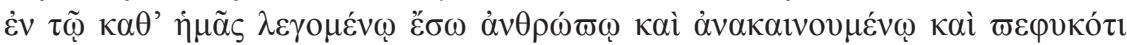

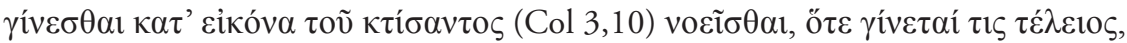

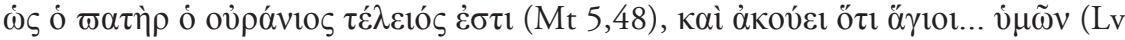

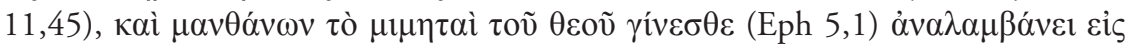

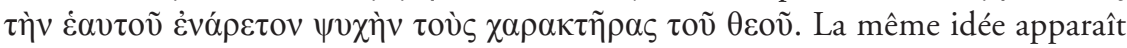

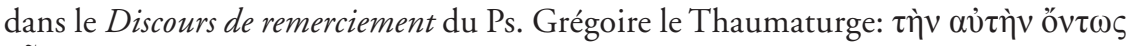
oṽ $\alpha \alpha v \theta \varepsilon \circ \tilde{~ \kappa \alpha i ̀ ~ \alpha ̉ v \theta \rho \omega ́ \varpi o v ~ a ̉ \rho \varepsilon \tau ท ́ v ~(P a n O r a t, ~ 142) . ~}$

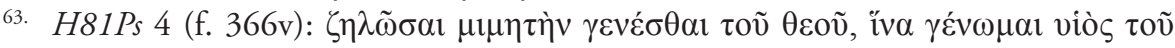

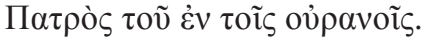

64. Le terme $\delta \omega \rho \varepsilon \alpha ́$ rappelle le motif de l'adoption filiale chez Irénée (Adv. Haer. III,19,1). Cf. Mosser, "The Earliest Patristic Interpretations of Psalm 82», cit., p. 47, à propos de Ps 81,7: "According to Irenaeus these words are spoken 'to those

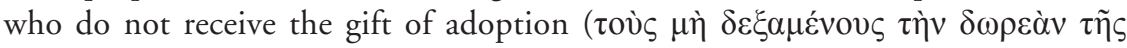


on pourrait remarquer que la 'divinisation' se présente comme un don de Dieu qui exige une réponse active de la part de l'homme; ou, en des termes semblables (du reste, rappelés par l'homélie elle-même), la 'divinisation' demande qu'on "mortifie les ouvres de la chair" $(\mathrm{Rm} 8,13)$ pour vivre sous le régime de l'esprit'.

Comme nous l'avons anticipé, évidemment l'explication du v. 1 se ressent de la proximité avec les vv. 6-7 ("Moi, j'avais dit: 'Vous êtes tous des dieux et des fils du Très Haut'. Mais vous mourez comme des hommes et vous tombez comme l'un des princes"). D'ailleurs le prédicateur les cite déjà expressément, avant de passer à l'apothéose' que subit l'âme, quand elle accueille le Logos divin. L'attention de l'Alexandrin se déplace par là des aspects plus immédiatement moraux et spirituels vers l'agent de la déification ainsi que vers son objet: en première formulation (grâce à l'allusion à Jn 10,35) (5) $^{6}$ 'est bien le Logos qui vient à l'âme, qui en fait par là un 'dieu'. Avec ces considérations nous entrons dans un ordre d'idéee différent - encore qu'il soit complémentaire au précédent -, qu'on pourrait nommer par commodité le 'processus physique' ou 'anthropologique' de la déification. Une définition pareille n'est pas du tout déplacée, puisqu'Origène se sert de la parabole du levain $(\mathrm{Mt}$ 13,33; Lc 13,20-21), qui entraîne la représentation d'une transformation de la réalité. Or l'Alexandrin - selon une exégèse qui ne réapparaît, en de termes proches, que dans un fragment sur Luc (s'il lui revient) -, à la suite de $1 \mathrm{Th} 5,23$, interprète les "trois mesures de farine", qui reçoivent le levain, comme les trois composantes de l'homme: esprit, âme et chair. La femme est l'Église qui accueille le Christ et insère son 'levain' dans l'homme, afin qu'il devienne tout entier dieu ${ }^{66}$. À la différence de notre homélie, le fragment sur Luc identifie le 'levain' à l'Esprit-Saint,

vio $\theta \varepsilon \sigma i \alpha \varsigma)$, but who despise the incarnation of the pure generation of the Word of God, depriving man of ascent to God".

65. On relève une citation du passage johannique en $H 81 P s$ s (f. 369r).

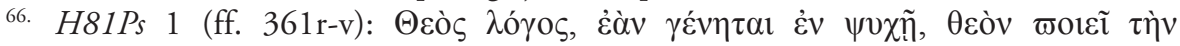

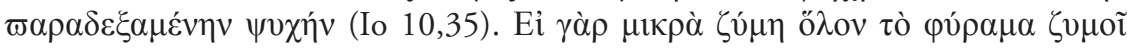

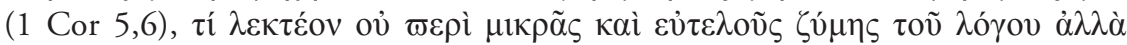

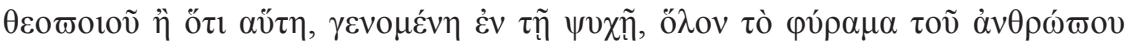

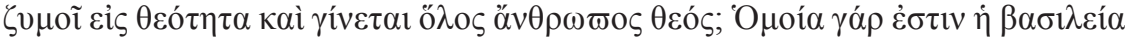

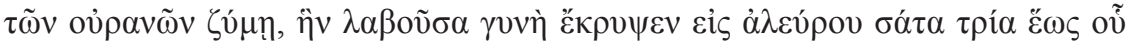

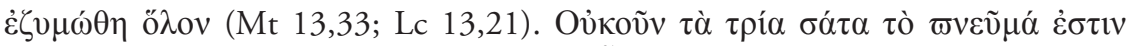

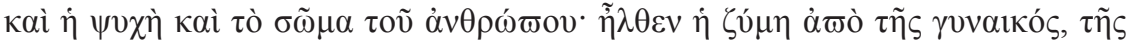

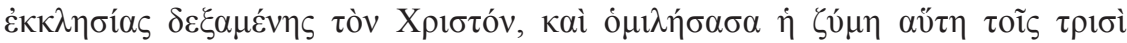


tandis que notre sermon le rapporte au Logos, qualifié du reste comme 'déificateur' ( $\theta \varepsilon 0 \varpi 0 \imath o ́ s)$. En outre, tandis que l'homélie aboutit à l'affirmation de la déification, le fragment parle plutôt de la 'sanctification' mise en acte par l'Esprit ${ }^{67}$.

Mais ce qui est plus frappant ici, au dire même du prédicateur, est le fait de considérer les effets du processus de la déification sur les trois composantes de l'homme. Il observe, en effet, que ce n'est pas surprenant

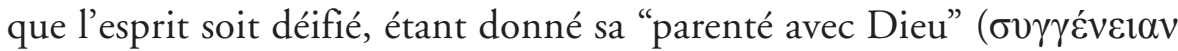

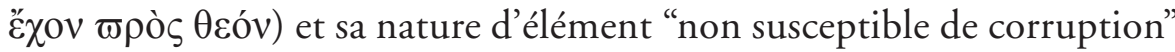
$(\alpha \not \varphi \theta \alpha \rho \tau o v)$ dans chaque homme, ce qui vraisemblablement renvoie à l'esprit comme le siège de l'image de Dieu dans l'homme. Par contraste, on peut parler en quelque mesure de 'surprise' à propos de l'âme, du fait qu'elle, à cause du libre arbitre, est sujette à pécher et dès lors à mourir, selon la référence très courante chez Origène à Ez 18,4 ("L'âme qui pèche mourra"): précisément en raison de sa peccabilité, la déification intervient afin qu'elle ne pèche plus et ne meure plus. Mais "ce

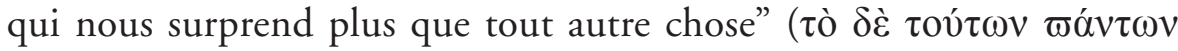
$\theta \alpha v \mu \alpha \sigma i \omega ́ \tau \varepsilon \rho o v)$ est la déification du corps: elle opère aussi sur celui-ci, pour qu'il ne soit plus "chair et sang" (1 Cor 15,50), mais devienne "conforme au corps glorieux" du Christ (Phil 3,21) et, étant ainsi déifié, il monte lui aussi vers le ciel parvenant à sièger avec Dieu dans l'assemblée des 'dieux' ${ }^{68}$. La déification du corps trouve par là son prototype et

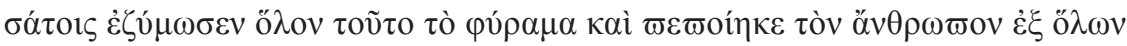

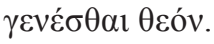

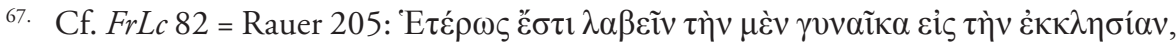

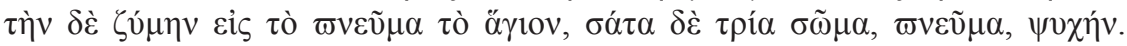

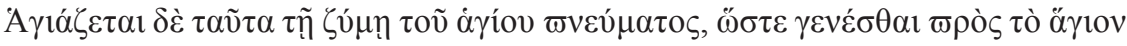

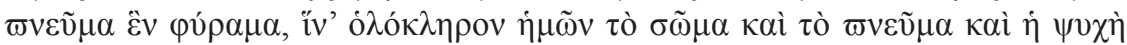

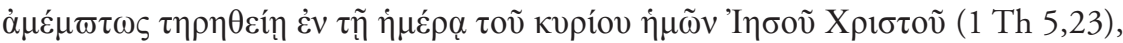

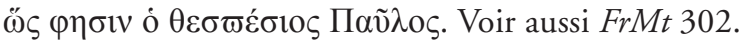

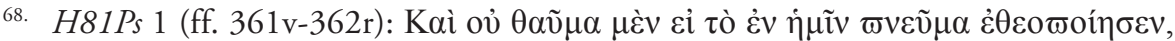

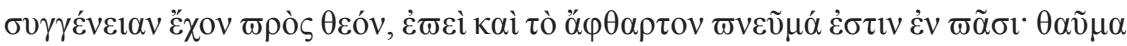

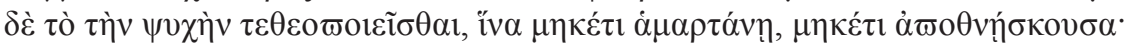

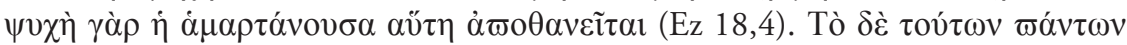

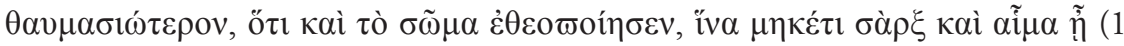

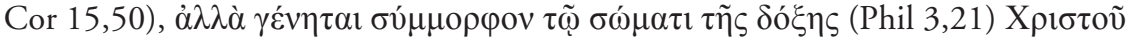

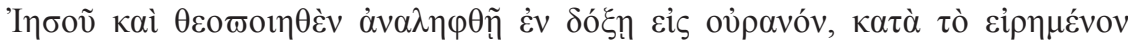

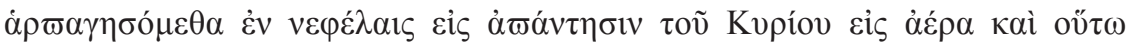

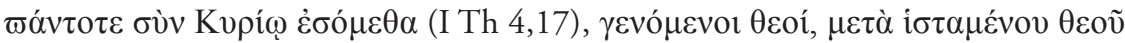

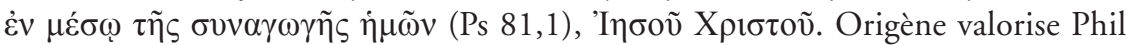


son modèle dans le corps glorieux du Christ et nous rappelle l'exposé donné à ce propos dans la 2ème Homélie sur le Psaume 15.

Celle-ci a attiré l'attention de Pamphile, qui en a repris un passage important dans son Apologie d'Origène, en vue de le défendre de l'accusation de nier la résurrection de la chair ${ }^{69}$. Dans cette homélie, qui insiste particulièrement sur l'anthropologie tripartite pour tracer l'image du Christ comme étant lui-même un être 'composé' ( $\sigma u ́ v \theta \varepsilon \tau \tau o \zeta)^{70}$, l'Alexandrin repousse l'idée que le corps de Jésus était un corps 'pneumatique', 'de la même substance que le Logos', après avoir réaffirmé avec force la nature de 'corps terrestre' ( $\gamma$ ñivov $\sigma \tilde{\omega} \mu \alpha)$ chez le Ressuscitép1. Il développe à ce propos une exégèse du testimonium apostolique de Ps 15,9c ("Tu n'abandonneras pas mon âme dans l'Hadès"), invoqué par Pierre dans son discours le jour de Pentecôte (Act 2,25-28). D'autre part, cette exégèse s'appuie aussi sur Is 63 (vv. 1-3 et 7). De cette façon, le "Premier-Né des morts" (Col 1,18; Ap 1,5) est présenté par Origène comme celui qui monte triomphalement vers le ciel avec son corps, par une anabasis qui fait pendant à sa katabasis jusqu'à l'Hadès au moment de la mort. Jésus Christ est le premier homme à faire cette expérience, étant donné que selon notre homélie il n'est pas possible d'affirmer cela clai-

3,21 en liaison avec le thème de l'image du Logos, par ex., en HGn I,13: Quicumque ergo veniunt ad eum et rationabilis imaginis participes effici student, per profectum suum secundum interiorem hominem renovantur cotidie (2 Cor 4,16) ad imaginem eius, qui fecit eos; ita ut possint conformes corpori claritatis eius (Phil 3,21) effici, sed unusquisque pro viribus suis.

69. Pamph., Apol. 142-145.

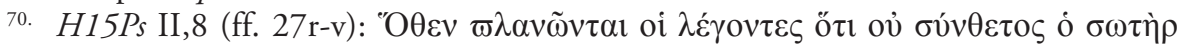

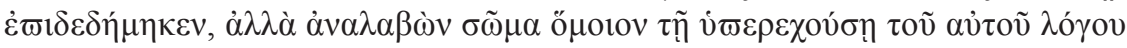

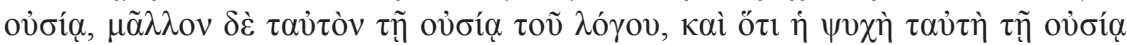

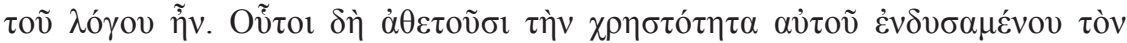

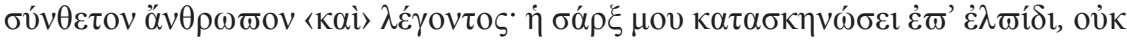

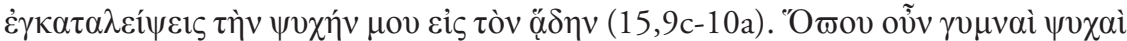

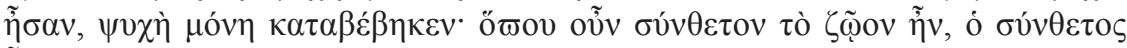
$\tilde{\eta} \lambda \theta \varepsilon \mu \varepsilon \tau \dot{\alpha} \psi v \chi \tilde{\eta} s$. La polémique contre les tenants d'un 'corps pneumatique'

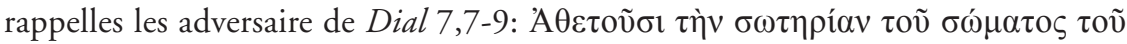

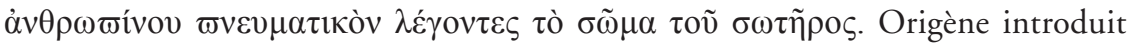

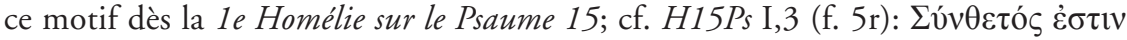

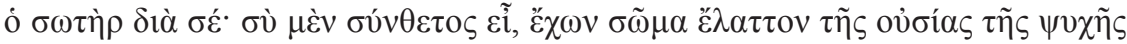

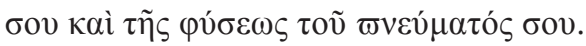

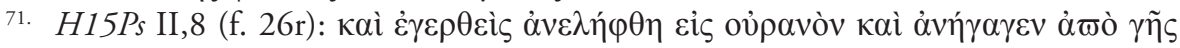

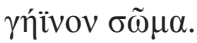


rement pour Hénoch ou pour Élie, à partir du témoignage scripturaire à leur égard ${ }^{72}$. Mais le corps du Ressuscité apparaît comme un corps encore marqué par "les traces du sang et des blessures", car les puissances angéliques, qui pour Origène engagent un dialogue direct avec le Christ sur la base d'Is 63, s'émerveillent de la rougeur de ses vêtements.

Par conséquent, l'ascension glorieuse du Christ au ciel dans son corps ne peut que constituer le paradigme exemplaire pour la déification du corps de l'homme, si on laisse de côté en ce moment les problèmes que l'exposé de la $2 e$ Homélie sur le Psaume 15 soulève avec son insistance sur la nature 'terrestre' et non 'pneumatique' du corps du Ressuscité ${ }^{73}$. D'autre part, l'idée que le Christ en tant qu'homme, par le fait de ressusciter arrive à être 'déifié', revient ailleurs chez Origène (non seulement dans les homélies de Munich) et nous laisse entrevoir la réponse qu'il faut donner à l'interrogation sur la divinisation du corps. Comme le dit la 15e Homélie sur Jérémie, unissant les deux aspects principaux que notre analyse de l'Homélie sur le Psaume 81 vient de mettre en évidence:

Moi-même, à cause de lui, je ne suis plus un homme, si j'observe ses paroles, mais il affirme: "Moi, j'ai dit: vous êtes tous des dieux et des fils du Très Haut" (Ps 81,6). Donc, tout comme il est "Premier-Né d'entre les morts" (Col 1,18; Ap 1,5), il est devenu "Premier-Né" de tous les hommes en se transformant en $\mathrm{Dieu}^{74}$.

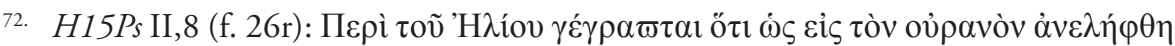

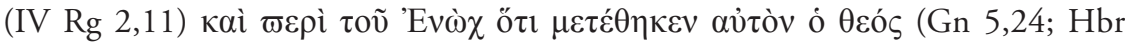

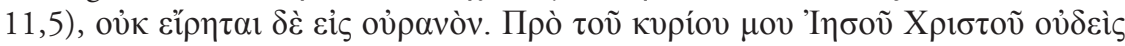

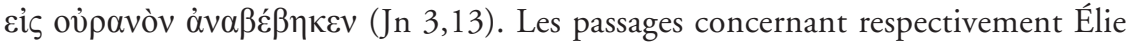
(CIo VI,11,71; VI,13,77) ou Hénoch ( $F r G n, 648$ Petit) ne semblent impliquer non plus une reconnaissance explicite de l'ascension de leur corps au ciel.

73. Voir, à ce propos, G. Sfameni Gasparro, Art. "Corpo», in A. Monaci Castagno (dir.), Origene. Dizionario: la cultura, il pensiero, le opere, Roma, 2000, p. 87-92. L'A. rappelle, à juste titre, les tension et les apories de la doctrine de l'Alexandrin sur le corps. Cf. aussi E. Prinzivalli, Art. «Resurrezione», ibid., p. 401-405, spécialement la page 404.

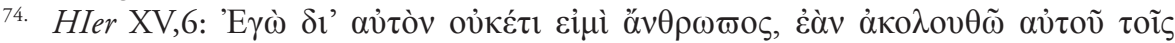

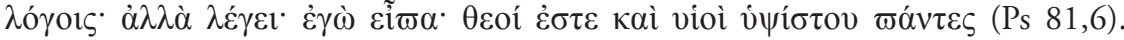

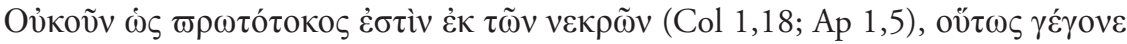

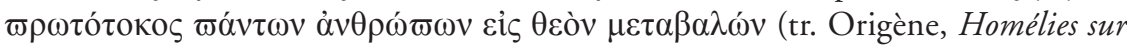
Jérémie, tr. par P. Husson† et P. Nautin, éd. P. Nautin, SC 238, Paris,1977, p. 127). 
Cette explication rejoint la solution donnée par Origène à une objection fictive dans la 1e Homélie sur le Psaume 67, alors qu'il interprète

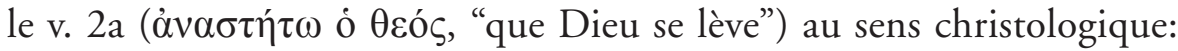
si le Sauveur, qui est Dieu, resurgit, faut-il dire que 'Dieu est mort'75? La réponse exploite l'idée paulinienne de la transformation de l'élément 'psychique' en 'pneumatique' selon 1 Cor 15, le passage auquel l'Alexandrin recourt régulièrement afin d'élaborer sa doctrine de la résurrection des corps. Donc, ce qui meurt dans le Christ c'est l'homme; ce qui resurgit, ce n'est plus l'homme mortel, mais bien 'dieu'76. La 'divinisation' du Ressuscité consiste précisément dans son immortalité, qui est par principe un attribut exclusif de Dieu, comme Origène le rappelle souvent à la suite de 1 Tim 6,16 ("le seul qui possède l'immortalité") ${ }^{77}$. Notre homélie converge alors avec le passage du 2e Livre du Contre Celse, où l'Alexandrin déclare la mortalité comme trait constitutif de Jésus en tant qu'homme en s'appuyant sur Rm 6,9 ("le Christ une fois ressuscité des morts ne meurt plus... la mort n'exerce plus de pouvoir sur lui”" $)^{78}$.

Ce n'est peut-être pas un hasard si Origène poursuivant dans l'Homélie sur le Psaume 81 l'idée de la déification à la lumière du v. 1, évoque encore la résurrection des morts en relation à 1 Cor 15,41, quoiqu'il utilise ce passage surtout pour indiquer le jugement circonstancié de Dieu et à sa suite les différentes destinées eschatologiques de ceux qui sont des 'dieux', avec une référence explicite aux anges ${ }^{79}$. Mais l'Alexandrin

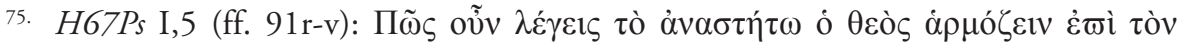

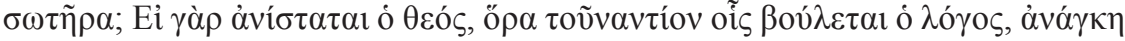

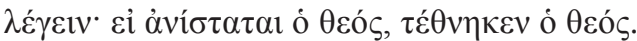

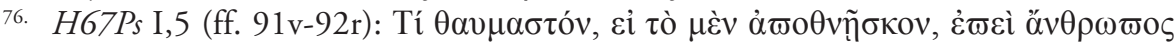

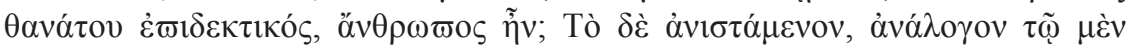

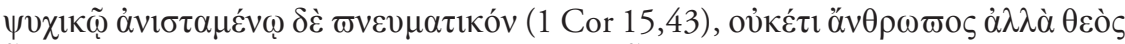

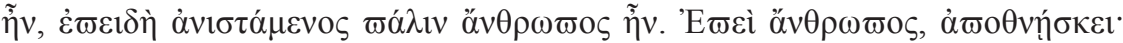

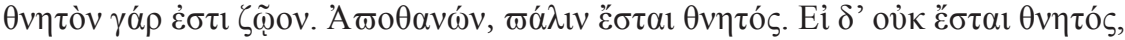

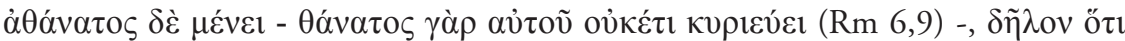

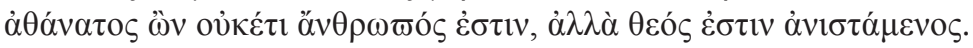

77. H15Ps I,9 (f. 15v); H77Ps IV,7 (f. 255r). Voir aussi CIo II,17,123.

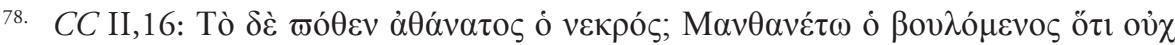

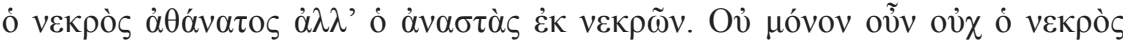

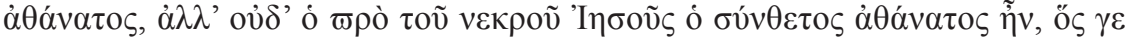

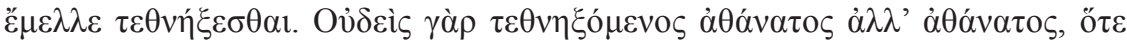

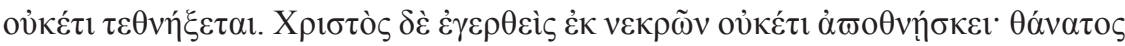

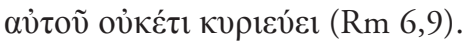

79. Cf. H81Ps 2 (ff. 362r-v). 
revient après cela à la note qui avait introduit le thème de la divinisation dans le prologue: ce qui s'oppose à la condition de 'dieux' est le péché des fidèles en tant qu'hommes. Il critique à ce moment les 'distinctions' accordées aux riches au sein de la communauté ecclésiale dans un passage qui exploite les diverses significations de l'expression du v. 2: ఐрó $\sigma \omega \varpi \alpha$ $\lambda \alpha \mu \beta \alpha ́ v \varepsilon \varepsilon v$, "faire des distinctions" mais aussi "assumer des rôles" au sens de 'visages', 'masques' ou 'personnes'. L'Alexandrin rappelle que si les anges sont en mesure d'"assumer le visage de Dieu", même les fidèles sont invités à le prendre sur eux-mêmes, se faisant ses imitateurs et devenant par l'aide aux plus démunis des vrais 'fils de Dieu'. Cela prélude à l'appel à la sanctification adressé à tous les fidèles qu'Origène tire du v. 6. Par une de ces prosopopées qui sont si chères à Origène, le peuple de Dieu dans son ensemble est appelé à 'devenir dieu' et pas seulement les évêques, les prêtres et les diacres ${ }^{80}$.

\section{V. ÉPILOGUE: UNE PERSPECTIVE BIBLIQUE ET SOTÉRIOLOGIQUE}

Au terme de notre analyse une considération s'impose: le thème de la déification s'avère être pour Origène de nature assez complexe, sinon en quelque mesure omnicompréhensive. Nous nous sommes efforcés de le revisiter à la lumière de plusieurs perspectives - terminologique, scripturaire, exégétique et théologique -, d'ailleurs étroitement liées l'une à l'autre, sans parvenir pour autant à en mesurer toute la profondeur. En raison de sa retombée plus vaste, il faut reconnaître que la doctrine de la déification se manifeste sous plusieurs facettes. Sans vouloir maintenant les résumer dans une formule inévitablement trop appauvrie, nous avons quand même l'impression que le thème de la déification s'articule pour l'Alexandrin à l'intérieur d'un discours primairement biblique et sotériologique, sans que nous réussissions à éclairer plus directement toutes ses implications ontologiques. En effet, ce discours trouve son axe principal dans le rôle médiateur du Logos à travers la création et l'œuvre de la rédemption (impliquant aussi la coopération de l'Esprit, encore qu'elle soit restée un peu à l'ombre dans notre dossier).

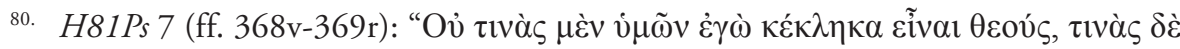

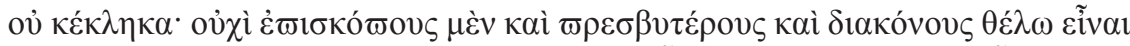

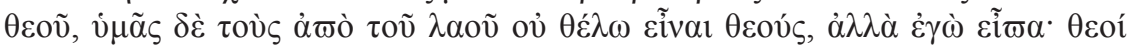

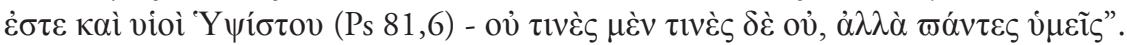


Dans les textes que nous avons examinés, la visée, pour ainsi dire, 'physique' de la déification ne joue presque jamais un rôle déterminant de même que sa résolution 'mystique', car seul le Christ est assuré d'une participation distinctive à la divinité du Père, parce qu'il le contemple continuellement. Le caractère unique de la 'divinisation' du Christ en relation au Père est bien mis en relief dans les nouvelles Homélies sur les Psaumes. En effet, comme le dit la 1e Homélie sur le Psaume 15, le Christ qui 'adorne' tous les êtres spirituels, en venant dans le monde pour réaliser leur accomplissement, a été lui-même 'adorné' par le Père, "devenant Dieu" à partir de lui ${ }^{81}$. De même, la contemplation du visage du Père est, selon la 2e Homélie sur le Psaume 15, une perpétuelle source de joie pour le Fils ${ }^{82}$, ce qui nous rappelle l'approche, pour ainsi dire, 'iconique' de la déification dans le face-à-face avec le Logos que nous avons mis en évidence dans le Commentaire sur Jean et le Traité sur la prière ${ }^{83}$. Sur le fondement du rapport privilégié entre le Père et le Fils, nous entrevoyons aussi le thème de la nourriture spirituelle qui entretient dynamiquement leur relation contemplative, tandis qu'elle représente le modèle pour toute vie spirituelle des hommes et, par conséquent, pour leur engagement dans le procès de la déification aboutissant également à une contemplation divinisatrice ${ }^{84}$.

Réunissant des regards divers qui convergent tous vers le déroulement de l'histoire du salut, la déification apparaît au départ comme l'inscription primordiale de la 'vocation divine' dans l'homme en tant qu'être créé “à l'image de Dieu”. Ayant déchu de cette condition ori-

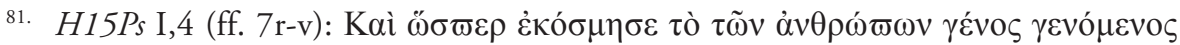

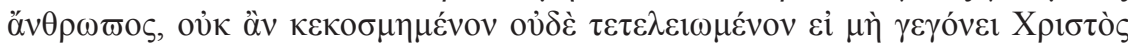

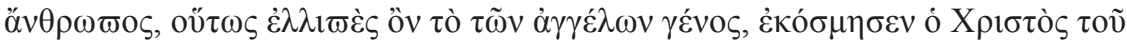

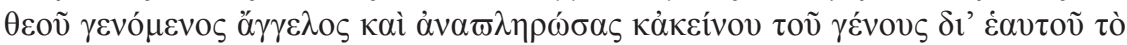

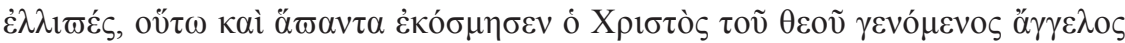

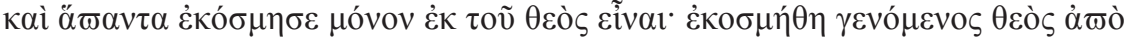

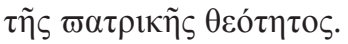

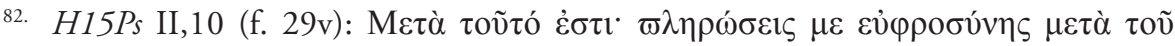

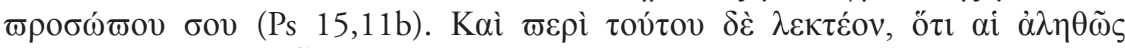

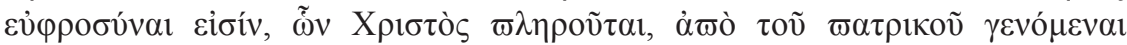

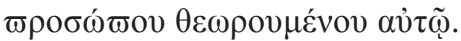

83. Cf. supra notes 13 (CIo XXXII,27,338-339) et 14 (Orat XXV,2). Sur ce sujet, voir Bruns, Trinität und Kosmos. Zur Gotteslehre des Origenes, cit., p. 288-300 ("Vergöttlichung in der Schau des Vaters"). Voir aussi CIo I,16,92.

84. Le texte fondamental est H77Ps IV, mais le motifs des aliments spirituels est très répandu dans le Cod. Gr. 314 comme dans les autres écrits d'Origène. 
ginaire de participation à la divinité, à cause de son péché, l'homme est attiré à nouveau vers Dieu par l'intervention salvifique du Christ dans l'histoire. Celle-ci renouvelle ainsi l'appel à l'imitation de Dieu en sa perfection et sainteté, déjà annoncé dans l'Ancien Testament, tandis qu'elle aide à réaliser pleinement la ressemblance avec Dieu présente en germe dans l'homme en tant qu'image du Logos. Une pareille dynamique implique en somme la conformation au Christ en tant que Dieu fait homme, sans que la différence ontologique entre l'homme et Dieu soit jamais annullée. La divinisation de l'homme, engageant aussi son corps selon le modèle archétypique du Christ ressuscité, débouche finalement sur l'immortalité et l'incorruption retrouvées autant que sur la pleine acquisition de la condition de fils adoptifs de Dieu. En ce sens, comme le dit le Contre Celse, l'économie du salut accomplit graduellement la communion entre Dieu et l'homme, car elle parvient à "entrelacer la nature divine avec la nature humaine" et à rendre celle-ci "divine" non seulement dans le Christ, mais aussi dans tous ceux qui accueillent son message $e^{85}$.

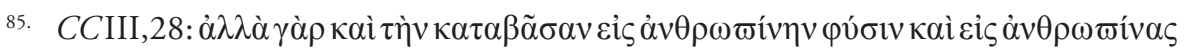

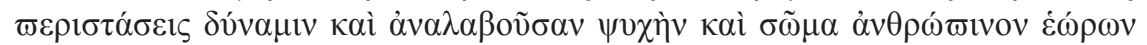

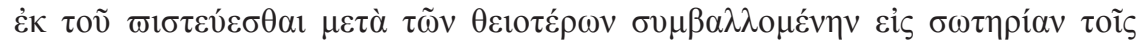

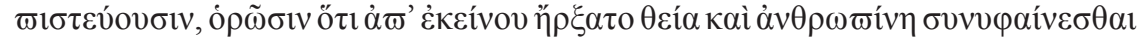

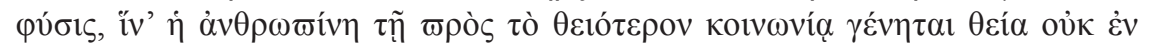

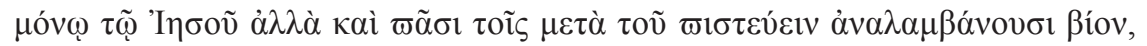

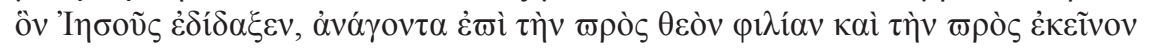

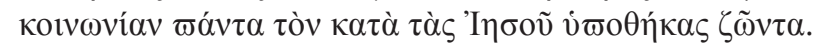

